\title{
Vortex Spinup Process in the Extratropical Transition of Hurricane Sandy (2012)
}

\author{
JUNG HOON SHIN \\ Department of Atmospheric and Oceanic Science, University of Maryland, College Park, \\ College Park, Maryland
}

(Manuscript received 24 April 2018, in final form 18 August 2019)

\begin{abstract}
This study utilizes the quasi-Lagrangian azimuthal momentum equation (i.e., budget calculation) and 1.667-km-resolution numerical simulation data to study the intensity and structural changes in Hurricane Sandy's extratropical transition. The results indicate that after the onset of extratropical transition, Sandy maintains an eyewall-like convection and warm core in the core region and has a frontal structure in the outer region. In the outer region, baroclinicity-driven frontal convection induces extensive planetary boundary layer (PBL) inflow, causing an inward advection of absolute angular momentum (AAM) per unit radius, which generates outer local wind maxima and expands Sandy's outer wind field through a spinup process. Moreover, because the outer tangential wind velocity accelerates in a frontal convection, local wind maxima associated with fronts can expand to the outer sides of frontal regions. Frontal convection increases AAM in the outer region, providing the precondition for reintensification; however, the front itself cannot cause Sandy's reintensification. The eyewall-like convection in the core region still plays an important role in Sandy's reintensification. When the baroclinic zone, where a strong horizontal temperature gradient exists, approaches the core region, the eyewall-like convection is enhanced because the warm, moist air of the core region is lifted by the cold, dry air associated with the approaching baroclinic zone. Consequently, owing to the enhancement of eyewall-like convection, the PBL inflow, which extends from the outer region to the core region, develops. This inflow increases the inward transportation of the outer frontal region's high-AAM air, thus leading to spinning up the core region's wind and reintensification.
\end{abstract}

\section{Introduction}

Along its track, Hurricane Sandy (2012) caused significant impacts from the Caribbean to the northeastern coastline of the United States (Blake et al. 2013). This hurricane underwent complex intensity and structural changes during its life, and became an anomalous large storm that reintensified before making landfall near New Jersey (Blake et al. 2013). Shin and Zhang (2017; hereafter denoted SZ17) have reasonably reproduced Sandy's intensity and structural changes by using version 3.4.1 of the Advanced Research version of the Weather Research and Forecasting (WRF) Model (ARW). They have concluded that (i) after 26 October, when extratropical transition (ET) began, three frontal areas of convection, $\mathrm{A}, \mathrm{B}$, and $\mathrm{C}$, successively occurred in the outer northeastern quadrant and propagated cyclonically, thereby causing the dramatic increase in Sandy's

\footnotetext{
Corresponding author: Jung Hoon Shin, ty9407@gmail.com
}

wind field, and (ii) deep convection in the core region played important roles in the reintensification.

Sandy evolved in a manner consistent with the ET process, because the storm interacted with cold, dry air (in the midlatitudes) when it moved toward the eastern coast of the United States (Blake et al. 2013; Galarneau et al. 2013; SZ17). ET is a process in which tropical cyclones (i.e., a warm core and nonfrontal cyclone) transform into midlatitude extratropical cyclones (i.e., a cold core and frontal baroclinic cyclone) (Klein et al. 2000). During the transformation stage of ET, tropical cyclones (TCs) usually weaken as the eyewall convection and warm core dissipate, and the frontal structure develops (Klein et al. 2000; Jones et al. 2003; Evans et al. 2006). Surprisingly, previous studies have found that some TCs (e.g., Hurricane Irene in 1999 and Hurricane Michael in 2000) intensified during the transformation stage when the hurricane had not completed ET (Abraham et al. 2004; Evans and Prater-Mayes 2004), and Sandy was one of those rare cases. However, most ET studies have focused on the reintensification process after ET was 
completed (Thorncroft and Jones 2000; Klein et al. 2002). Therefore, it is important to understand why some TCs reintensify under hostile conditions (e.g., strong vertical wind shear and the intrusion of cold, dry air) during the transformation stage of ET. Interestingly, Sandy shared features in common with other reintensifying storms (Irene in 1999, Michael in 2000): an eyewall-like convection $^{1}$ and warm core were maintained during this phase. Therefore, eyewall-like convection is likely to play an important role in the reintensification during ET, although Sandy became asymmetric, owing to strong vertical wind shear (VWS). Although a few studies have mentioned that an eyewall-like convection-driven warm core maintains low pressure in transforming hurricanes (Evans and Prater-Mayes 2004; SZ17), the intensification during the transformation stage and the impact of eyewall-like convection on such events have not been well examined.

Wind field expansion of TCs during ET is a challenging issue (Jones et al. 2003; Evans et al. 2017). Evans and Hart (2008) have studied the expansion of the wind field associated with Hurricane Bonnie (1998) and found that, in the ET event of Bonnie, the wind field (around 600-700-hPa layer) expanded, owing to the inward transport of absolute angular momentum (AAM) associated with the environmental conveyer belt flow. However, although (warm) frontogenesis is a major feature of ET (Jones et al. 2003), a detailed dynamic process associated with outer frontal convection that expands the wind field of TCs has not been published as a research article. It is widely accepted that the import of AAM within the planetary boundary layer (PBL) results in wind intensification of mature TCs because the eyewall convection induces PBL inflow (Yau et al. 2004; Smith et al. 2009; Smith and Montgomery 2015). Because a frontal convection develops in the outer regions of TCs that undergo ET, this outer convection generates $P B L$ inflow. This PBL inflow could transport AAM toward the outer convection, thereby intensifying or accelerating the cyclonic tangential velocity in the outer frontal regions of TCs (i.e., a spinup

\footnotetext{
${ }^{1}$ After 27 October, when Sandy was in the midlatitudes and exhibited the features of ET, the deep convection in the core region of Sandy did not encircle the eye by at least $180^{\circ}$ in both the observation and simulation. This deep convection does not satisfy the definition of an eyewall by the American Meteorological Society glossary; therefore, it is not an eyewall. However, this deep convection still (i) drives the secondary circulation of Sandy and (ii) maintains the storm's warm core (SZ17) and therefore plays a role similar to that of an eyewall. For this reason, in this study, the deep convection in the core region of Sandy is called an eyewall-like convection. This definition is applied after 27 October.
}

effect). If this effect occurs, the cyclonic circulation of TCs may expand because cyclonic circulation could also occur far from the center. On the basis of this concept, SZ17 has argued that the low-level wind field growth of Sandy is caused by the inward AAM advection due to the outer frontal convective regions. However, very detailed dynamic processes that expand the wind field of Sandy were not examined, and therefore, the influence of frontal convection on the wind field expansion of transforming TCs requires further study. Reintensification of TCs during the transformation stage and the wind field expansion are very important issues because they threaten the midlatitude region, where the population density is very high.

Because TC circulations tend to become highly asymmetric during ET (Merrill 1993; Fujibe and Kitabatake 2007; Evans et al. 2017), the present study applies asymmetric vortex spinup dynamics to the structural and intensity changes in the ET of Sandy. This asymmetric vortex spinup perspective is more detailed than SZ17 and has not been well examined in previous studies. The objectives of this study are to (i) investigate the spinup process and the expansion of the outer wind field due to frontogenesis (i.e., frontal convection) in the ET event of Sandy by using a budget calculation, (ii) analyze the detailed relation between the location of frontal convection and that of associated local wind maxima, and (iii) examine the important role of eyewall-like convection in the reintensification, all of which were not discussed in SZ17.

The next section reviews the evolution of Sandy on the basis of observations and the results of SZ17. Section 3 briefly introduces the azimuthal momentum equation of Yau et al. (2004), which will be used for the budget calculation in this study. Section 4 explains (i) how frontal convective regions generate local wind maxima in the outer region, expanding the areal size of the storm wind field through the inward advection of AAM per unit radius, and (ii) the relation between the local wind maximum location and frontal convection. Section 5 examines the spinup process during reintensification. The following section discusses how the fluctuation of eyewall-like convection affects the storm's intensity changes within the baroclinic zone where a strong horizontal temperature gradient exists. The last section provides a summary of this study.

\section{The life of Hurricane Sandy (observations and simulation)}

On the basis of SZ17 and the observed features of Sandy, the life of this hurricane can be divided into four stages, which are summarized in Table 1. On 24-25 October, Sandy was in the "rapid intensification (RI) 
TABLE 1. The life of Sandy, classified by different stages on the basis of the results of SZ17, observations, and features of ET. The ET onset stage (pre-reintensification stage) is almost equivalent to the weakening stage (steady-Vmax stage), as defined in SZ17.

\begin{tabular}{lcc}
\hline \multicolumn{1}{c}{ Stage } & Period & \multicolumn{1}{c}{ Major features and events } \\
\hline RI stage & $24-25$ Oct & $\begin{array}{c}\text { Sandy rapidly intensified as a major hurricane } \\
\text { Storm reached the first intensity peak (0600 UTC 25 Oct) } \\
\text { ET onset stage }\end{array}$ \\
Weakening and ET began as the storm entered the midlatitude region; one outer frontal \\
convective region \\
Frontal convection A developed in the northeastern quadrant of the storm around \\
0000 UTC 26 Oct
\end{tabular}

stage" and developed into a strong mature hurricane. During 25-26 October, Sandy entered the midlatitudes and began ET (ET onset stage). During this stage, the storm weakened due to strong VWS (Fig. 1d and SZ17). Figure 1a depicts that in this stage, a zonally elongatedfrontal convection band (A) developed to the northeast of Sandy, which was the sign of ET beginning. Between 27 and 28 October, during the "pre-reintensification stage," while the eyewall-like convection emerged in the core region, the number of outer frontal convective regions increased to two because of multiple frontogenesis events. As noted in SZ17, while the cyclonic circulation of Sandy continued to increase, the maximum wind speed of Sandy did not increase (i.e., no intensification) in this phase. Figure 1a illustrates that frontal convection A propagated cyclonically on 27 October, and finally the eyewall-like convection (denoted " $E$ " in Fig. 1a) emerged near the center as frontal convection A separated from the core region. As a result, ET was incomplete on 27 October, and this outer frontal convection and inner eyewall-like convection were maintained until Sandy made landfall in New Jersey (Blake et al. 2013). Around 0600 UTC 28 October, a new frontal convection B formed $\sim 300-400 \mathrm{~km}$ to the north-northeast of the eyewall-like convection. Six hours later, this frontal convection was also advected to the western semicircle, replacing the old frontal convection $\mathrm{A}$, and another frontal convection $\mathrm{C}$ then developed in the northeastern quadrant around 1800 UTC 28 October. Figure $1 \mathrm{~b}$ shows that this repeated frontal convection development and cyclonic propagation during the pre-reintensification stage are well-captured in the simulation of SZ17, although the frontal convection B is temporarily located closer to the storm center and penetrates deeper to the core region, as compared with observations around 0600 UTC 28 October. Except for this short period, the simulated frontal convection B exists in the outer region, similarly to the observations. However, Figs. 1a and 1b suggest that the observed fluctuation of core region convective activity, which can be roughly estimated on the basis of the areal size of the yellowshaded cold cloud top, is still well captured by the simulation. In the observations (simulation), the core region convective activity decreases after 1200 UTC (0600 UTC) 28 October as the frontal convection $\mathrm{B}$ moves to the western semicircle, thus suggesting that both exhibit similar fluctuations in a similar period. Additional model validation and storm features during the prereintensification stage are summarized in the appendix.

After 0000 UTC 29 October, Sandy reintensified (i.e., the increase of maximum wind speed or "reintensification stage") as the storm recurved northwest and approached the New Jersey coastline. Interestingly, as shown in Fig. 1c, the satellite image at 1800 UTC 29 October indicates that Sandy still maintained deep eyewall-like convection during the reintensification stage. Intense low-level winds exceeding $50 \mathrm{~m} \mathrm{~s}^{-1}$ were observed in the northwest of storm center around this time. Owing to the northwestward movement of the hurricane, the western baroclinic zone approached the core region, and SZ17 claimed that the merging processes between the eyewall-like convection and baroclinic region (and the eyewall-like convection itself) contributed to the reintensification. After the reintensification, the eyewall- 


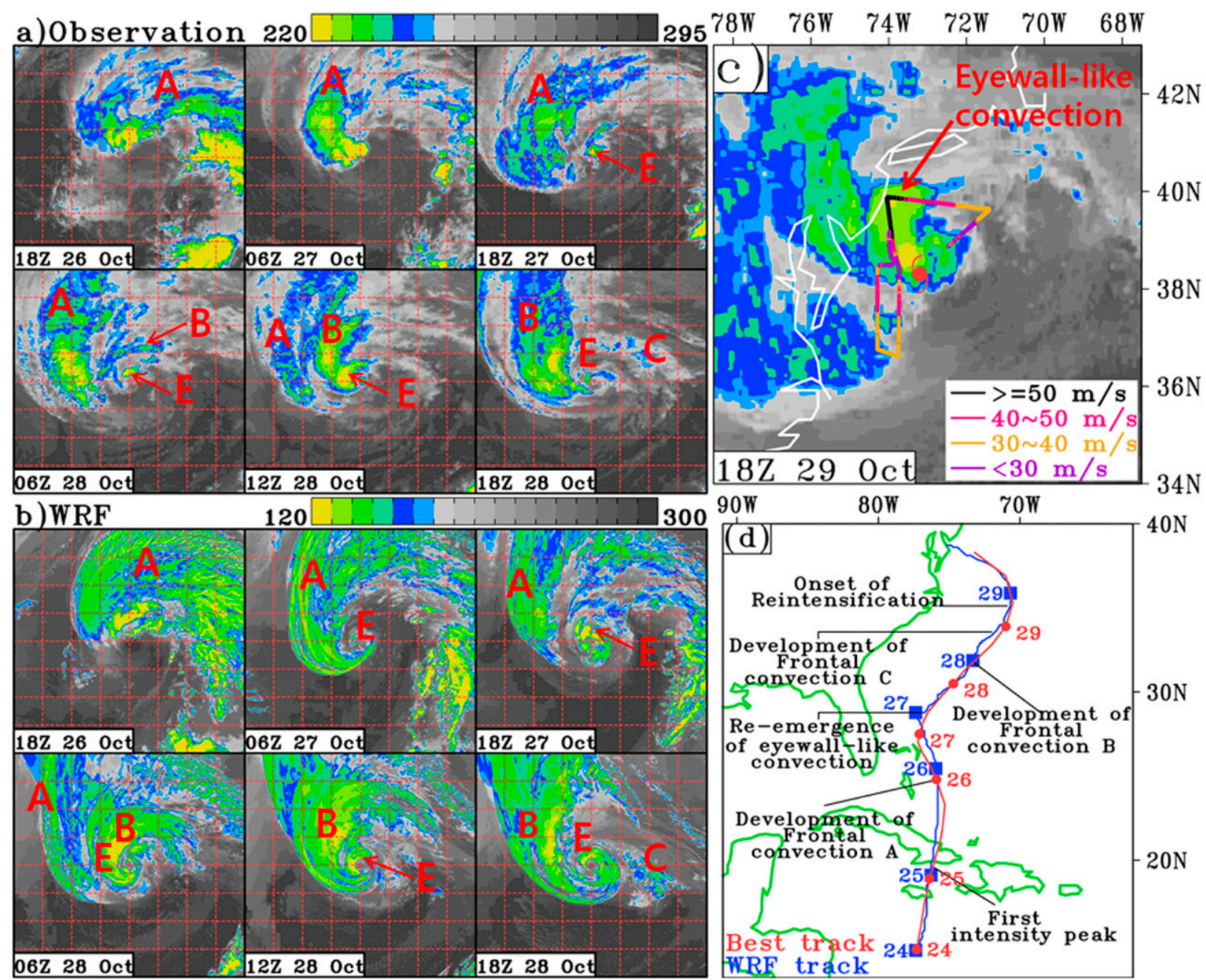

FIG. 1. (a) The cloud-top brightness temperature (shaded; 5-K interval) from the Gridded Satellite (GridSat-B1) data archive of the National Climatic Data Center of NOAA (http://www.ncdc.noaa.gov). Letters A, B, and C denote three frontal convective regions, and E indicates the eyewall-like feature. Roughly $20^{\circ} \times 20^{\circ}$ regions are shown with respect to the storm center in which each box indicates a $2.5^{\circ} \times 2.5^{\circ}$ area. (b) As in (a), but for simulated outgoing longwave radiation (shaded; $12 \mathrm{~W} \mathrm{~m}^{-2}$ interval) from 5-km-resolution domain of SZ17 simulation. (c) As in (a), but at 1800 UTC 29 Oct, superimposed with flight-level (mostly near 1.5-km level) wind speed (colored line; $\mathrm{m} \mathrm{s}^{-1}$ ) that was taken between 1800 and 2200 UTC on the same day during the flight mission 5308 2418A. Hurricane symbol indicates the storm center. (d) Best track (red) and simulated track (blue) of Hurricane Sandy between 0000 UTC 24 Oct and 1800 UTC 29 Oct. Hurricane positions at every 0000 UTC are marked as red circles and blue squares. The approximate positions where important events occur are denoted as black lines based on simulation and observation.

like convection dissipated, and thus Sandy completed ET (Blake et al. 2013).

\section{Methodology}

The WRF simulation of SZ17 is initialized at 0000 UTC 24 October and ends at 1800 UTC 29 October 2012 (hereafter day/hour format is used in figures, e.g., 29/18). The simulation uses (i) 45-, 15-, 5-, and 1.667-km-resolution domains, in which the two high-resolution domains are storm-following nests and (ii) a state-of-the-art TC vortex bogussing algorithm (Kwon and Cheong 2010;
Kwon et al. 2010). The physic parameterization options of the simulation are summarized in SZ17. In this study, the same model simulation as SZ17, but with 15-mininterval, $1.667-\mathrm{km}$-domain data, is utilized.

The momentum equation for azimuthal wind or tangential wind in cylindrical coordinates $(r, \lambda, z)$, which was used by Yau et al. (2004), is shown below in Eq. (1), where $U, V, W, \eta_{a}, p, \alpha, f, \phi, \Omega$, and FRI are radial velocity relative to Earth, tangential velocity relative to Earth, vertical velocity, absolute vorticity, pressure, specific volume, Coriolis parameter, latitude, Earth angular velocity, and friction plus diffusion, respectively. 
Details can be found in Yau et al. (2004). (In this study, the storm center, which is used for the cylindrical coordinate, is found at sea level and is defined as the geometric centroid of sea level isobars, following SZ17.) This momentum equation is

$$
\begin{aligned}
\left(\frac{\partial}{\partial t}+\frac{V}{r} \frac{\partial}{\partial \lambda}\right) V= & -\eta_{a} U-W \frac{\partial V}{\partial Z}+2 \Omega \cos \phi \sin \lambda W \\
& -\frac{\alpha}{r} \frac{\partial p}{\partial \lambda}+\text { FRI, } \quad \eta_{a}=\frac{1}{r} \frac{\partial r V}{\partial r}+f .
\end{aligned}
$$

An advantage of this equation is its capability to diagnose the asymmetric wind features of the hurricane, as demonstrated in the case of Hurricane Andrew (1992) by Yau et al. (2004). According to Yau et al. (2004), in this equation, the left-hand side [i.e., $\partial V / \partial t+(V / r)(\partial V / \partial \lambda)]$ is the quasi-Lagrangian tendency of tangential wind following the tangential flow (VTEN), and the right-hand side (i.e., forcing terms) consists of the radial absolute vorticity flux (RAF), vertical advection of tangential wind (VAT), Coriolis forcing (CF), pressure gradient in the azimuth direction (PGF), and friction plus diffusion (FRI). Here, (i) RAF is equivalent to the radial advection of $A A M$ per unit radius, and (ii) RAF plays a dominant role in generating positive VTEN (i.e., accelerating tangential wind velocity) near the surface, where PBL inflow transports angular momentum inward (Yau et al. 2004). To demonstrate how the eyewall-like convection and frontal convection spin up the vortex of Sandy, this study focuses on RAF within the PBL (i.e., $0.5-\mathrm{km}$ level), because convectively driven PBL inflow transports AAM inward, thus increasing cyclonic tangential wind velocity. For clear visualization, each term in Eq. (1) and the wind fields in the cylindrical coordinate $(r, \lambda, z)$ are smoothed 10 times by using a weighted smoother based on a circular diffraction weighting function in the Read Interpolate Plot (RIP; a standard graphic program for WRF) program subroutine. In addition, each term in Eq. (1) is smoothed into a 1-h running mean. FRI is not calculated, because the WRF output does not include this term.

To briefly validate the budget calculation regarding Eq. (1), Fig. 2 illustrates some example calculation results. Figures $2 \mathrm{a}-\mathrm{-e}$ compare the left-hand-side term (i.e., VTEN) and the summation of the first four forcing terms (i.e., RAF, VAT, CF, and PGF) on the right-hand side of Eq. (1) above PBL (where friction is negligible) at several time steps and different altitudes. Some differences between the left- and right-hand-side terms may be due to several computational procedures, for example, converting data into cylindrical coordinates (Abarca and Montgomery 2013) or numerical filtering (Leroux et al. 2013). Nonetheless, Figs. 2a-e indicate that, in the absence of friction, VTEN is similar to the summation of the four forcing terms, especially in the $50-550-\mathrm{km}$ radial annulus, thus suggesting that the budget calculation regarding Eq. (1) appears reasonable. In Fig. 2, the maximum tangential velocity is either collocated with the acceleration region or located slightly counterclockwise to the maximum acceleration region, because the tangential velocity accelerates (decelerates) where VTEN is positive (negative).

\section{The impact of the front-driven spinup process on storm size expansion}

As shown in Fig. 1, because the outer frontal convection developed continuously after 26 October, Sandy accompanied the frontal convection in the midlatitudes. By using Eq. (1), several factors are investigated, including that (i) the outer frontal convection generates intense PBL inflow that blows toward these convective regions, and (ii) this effect accelerates and induces cyclonic tangential flow in the outer frontal ${ }^{2}$ region, thereby expanding the cyclonic circulation of Sandy through the spinup effect. To explain these issues, the structural evolution of the PBL (i.e., $0.5-\mathrm{km}$ level) between RI and the pre-reintensification stage is displayed in Fig. 3. Model integration times, including the first intensity peak (0200 UTC 25 October; RI stage), the development of the first frontal convection A (0000 UTC 26 October; ET onset stage), the emergence of eyewalllike convection and the cyclonic propagation of frontal convection A (0600 UTC 27 October; early phase of the pre-reintensification stage), and the development of the second frontal convection B (0000 UTC 28 October; the middle of the pre-reintensification stage), are discussed in this section.

Figures $3 \mathrm{a}$ and $3 \mathrm{e}$ show that at 0200 UTC 25 October, in high-equivalent-potential-temperature $\left(\theta_{e}\right)^{3}$ tropical air, Sandy reaches its first maximum intensity after RI, exhibiting a well-defined eyewall and a small $(\sim 50-\mathrm{km}$ radius) radius of maximum wind (RMW) (SZ17). Owing

\footnotetext{
${ }^{2}$ In this study, the core region is within an approximately $200-\mathrm{km}$ radius because Sandy is an anomalously large storm, and convective cells associated with the eyewall-like convection are mostly spread within a $200-\mathrm{km}$ radius. For this reason, the outer region in this study is outside the $\sim 200-\mathrm{km}$ radius. The reference point of the "radius" is the storm center, and "inflow" in this paper refers to the low-level airflow that blows into convection associated with Sandy (i.e., frontal convection, eyewall, or eyewall-like convection).

${ }^{3}$ In this study, the frontogenesis function $\left(\theta_{e}\right)$ fields are smoothed 30 (20) times with a weighted smoother in the RIP program. The scalar frontogenesis function follows Eq. (2.6a) of Keyser et al. (1988).
} 


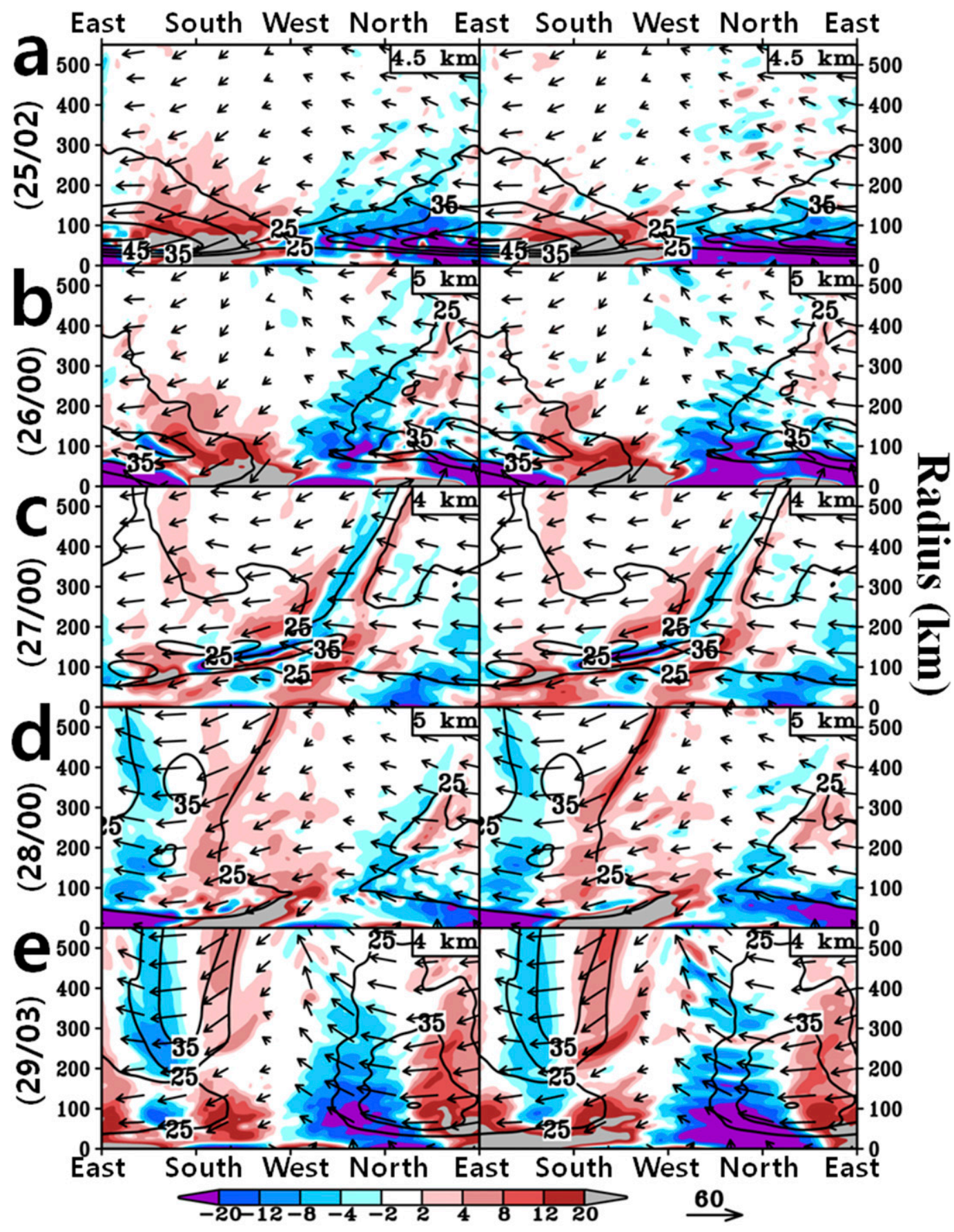

FIG. 2. Radius-azimuth cross section of (left) the quasi-Lagrangian tendency of tangential wind following the tangential flow and (right) the summation of the right-hand-side terms in the azimuthal momentum equation, excluding friction and diffusion, (shaded; $\mathrm{m} \mathrm{s}^{-1} \mathrm{~h}^{-1}$ ) at (a) 25/02 (i.e., 0200 UTC 25 Oct), (b) 26/00, (c) $27 / 00$, (d) $28 / 00$, and (e) $29 / 03$. Tangential velocity (black contours; $10 \mathrm{~m} \mathrm{~s}^{-1}$ interval above $25 \mathrm{~m} \mathrm{~s}^{-1}$ ); horizontal (i.e., tangential and radial) flow vectors are superimposed. Vertical level $(\mathrm{km})$ is given in the upperright corner. The vertical axis indicates the distance $(\mathrm{km})$ from the storm center. All horizontal flows are relative to Earth, following Yau et al. (2004), all fields are smoothed into a 1-h running mean and, data from the WRF 1.667-km-resolution domain are used, which are the same for all following figures, unless otherwise mentioned. 


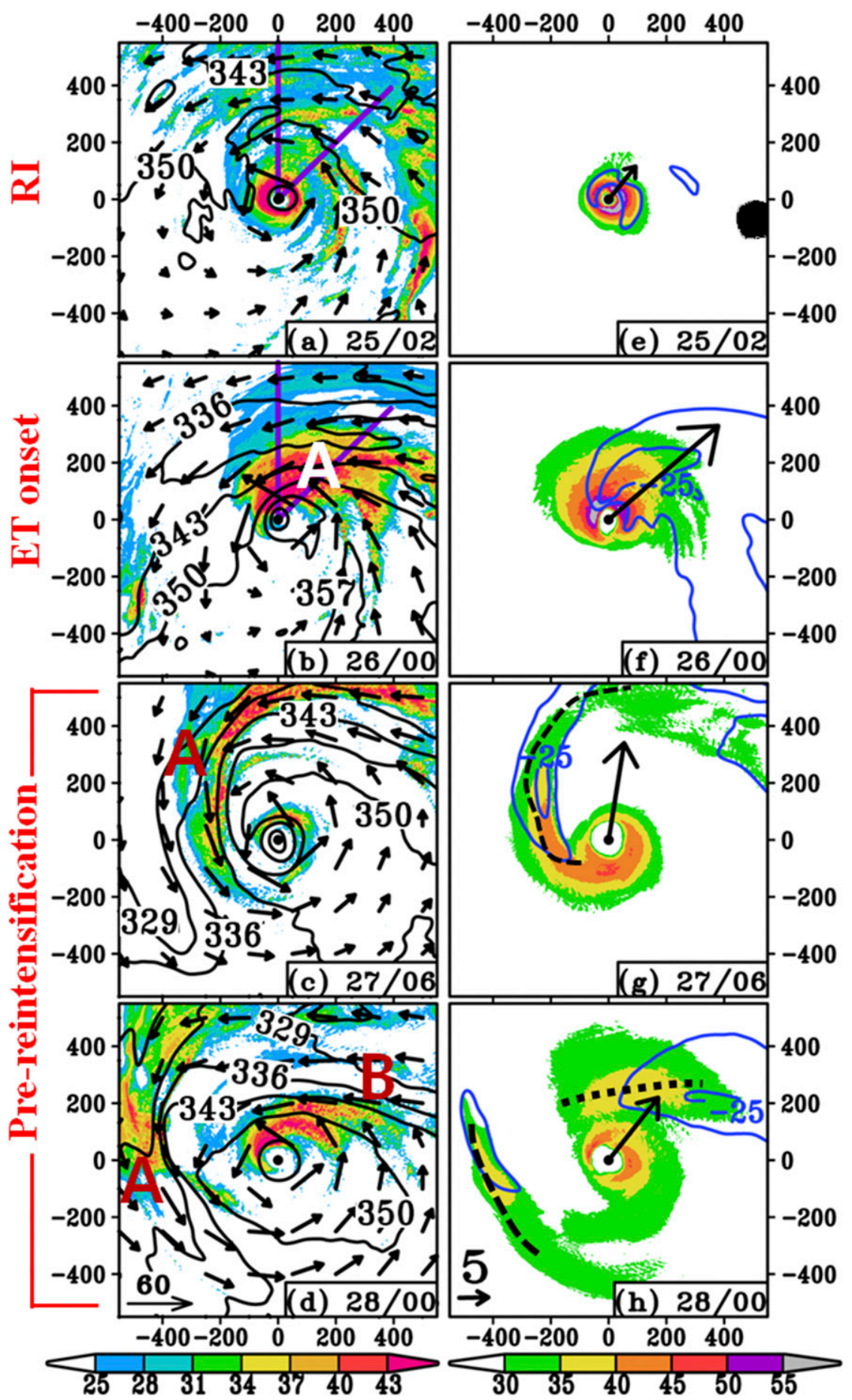

FIG. 3. (a)-(d) Horizontal structure of composite radar reflectivity (shaded; dBZ), equivalent potential temperature (contours; $7-\mathrm{K}$ interval), and horizontal flow vectors at the $0.5-\mathrm{km}$ level at 25/02, 26/00,27/06, and 28/00, respectively. Frontal convective and frontogenetic regions are indicated as A and B. (e)-(h) As in (a)-(d), but for inward radial velocity (blue contours at -15 , and $-25 \mathrm{~m} \mathrm{~s}^{-1}$ ), horizontal wind speed (shaded; $\mathrm{m} \mathrm{s}^{-1}$ ), and the 200-850-hPa vertical wind shear (VWS) vector. The scaling vector for VWS is shown in (h). The axis of local wind maximum associated with frontal convection is roughly indicated by the dotted or dashed line. Horizontal and vertical axes show the distance $(\mathrm{km})$ from the hurricane center (black dot). Inward radial velocity is smoothed 20 times using a weighted smoother in the RIP program. 
to a low VWS, ${ }^{4}$ the storm has an almost symmetric structure, and a strong PBL inflow that exceeds $15 \mathrm{~m} \mathrm{~s}^{-1}$ is mostly located near the RMW. This is a common feature of mature hurricanes (Yau et al. 2004; Bui et al. 2009). Around 0000 UTC 26 October (Figs. 3b,f), ET is initiated as Sandy moves northward and interacts with cold, dry (i.e., low- $\theta_{e}$ ) air (SZ17). As a result, frontal convection A develops in the northeast quadrant, owing to confluence, as shown in Fig. $5 \mathrm{i}$ of SZ17. In response to frontogenesis, a local wind maximum and a vast area of intense PBL inflow are generated near the frontal convection $A$ and in the eastern semicircle, respectively. As will be shown later, this broad, intense inflow, driven by frontal convective regions, spins up the outer region of Sandy through the inward RAF, and expands the cyclonic circulation of this storm. Thirty hours later (Figs. 3c,g; pre-reintensification stage), as the frontal convection A is advected cyclonically (SZ17), the associated intense PBL inflow (local wind maximum near the center) propagates to the northwestern (southwestern) quadrant. Around 0600 UTC 27 October, frontal convection A (i.e., baroclinic zone) is detached from the core region and propagates to the outer region at 0000 UTC 28 October (Figs. 3d,h), thereby delaying ET completion $^{5}$ during the pre-reintensification stage, as shown in Fig. 1a. As a result, the eyewall-like convection becomes isolated on 27 October and a warm core is maintained until the reintensification stage. While this frontal convection generates narrow and elongated local wind maximum exceeding $30 \mathrm{~m} \mathrm{~s}^{-1}$ in the western semicircle, another area of frontal convection B develops in the northeastern quadrant as a result of confluence between southerly and easterly winds, as shown in Fig. 3d herein and Figs. 6d and 6k of SZ17. This frontal convection induces another local wind maximum north of the storm center. Because the simulated frontal convection B exists closer to the storm center than shown in the observations (Fig. 1a), the associated local wind maximum (and baroclinic zone) is also temporarily located close to the center at this time. After around 0900 UTC 28 October, they move back to the observed

\footnotetext{
${ }^{4}$ VWS is obtained from SZ17 and is calculated from a $1000 \mathrm{~km} \times$ $1000 \mathrm{~km}$ (with respect to the storm center) areal-averaged wind velocity difference between 200 and $850 \mathrm{hPa}$.

${ }^{5} \mathrm{ET}$ is completed when the low-level baroclinic zone in the outer region reaches the storm center (Klein et al. 2000). Therefore, if it propagates away from the storm center, ET completion is delayed or halted. The baroclinic zone indicates where a strong horizontal temperature gradient exists, and the frontal convective region is the area where convection occurs along this baroclinic zone. In this study, these two regions almost coincide because frontal convection occurs in a significant portion of the baroclinic zone.
}

position (Fig. 6). Now, because Sandy has two regions of outer frontal convection, two intense PBL inflow areas exist near the two frontal regions, as shown by dark blue contours in Fig. $3 \mathrm{~h}$. Figures $3 \mathrm{~d}$ and $3 \mathrm{~h}$ reveal an interesting relation between the location of frontal convection $\mathrm{A}$ and the associated local wind maximum area, which was not discussed in detail in SZ17. A local wind maximum, where the wind speed exceeds $30 \mathrm{~m} \mathrm{~s}^{-1}$, not only exists in the frontal convective region but also often extends outside of this region (i.e., the downstream side of frontal convection). Such a relationship between the local wind maximum and frontal convective regions is also prominent, especially in the southern semicircle, after 0000 UTC 28 October (Figs. 6k,1,m of SZ17 and Figs. 6f,g herein) and will be explained further through the budget calculation.

To examine the role of the inward advection of AAM per unit radius (i.e., RAF) in the spinup process of the outer regions of Sandy, the quasi-Lagrangian tendency of tangential velocity (VTEN) and RAF at $0.5-\mathrm{km}$ level are shown in Fig. 4. Figures 4a and 4e show that before Sandy enters the transformation stage of ET (at 0200 UTC 25 October), intense tangential flow $\left(>25 \mathrm{~m} \mathrm{~s}^{-1}\right)$, the positive VTEN region, and strong RAF $\left(>8 \mathrm{~m} \mathrm{~s}^{-1} \mathrm{~h}^{-1}\right)$ exist mostly within a $150-\mathrm{km}$ radius. In particular, Fig. $4 \mathrm{e}$ depicts that a positive RAF region exists in the eastern semicircle, in agreement with the PBL inflow region. The outward bulge of the $25 \mathrm{~m} \mathrm{~s}^{-1}$ contour in the southeastern and northeastern quadrant implies the expanding of cyclonic circulation due to RAF, but the expansion is not yet noticeable. However, the size and magnitude of the positive VTEN region grows dramatically in the north and southeast of the center when ET is initiated (Fig. 4b). In agreement with this pattern, during the ET onset stage, the cyclonic circulation of Sandy expands, especially in the northern semicircle. As shown in Fig. 4f, the PBL inflow grows in size and magnitude (i.e., the radial inflow exceeds $20 \mathrm{~m} \mathrm{~s}^{-1}$ ) mostly in the eastern semicircle, thus increasing the inward RAF. The PBL inflow and RAF are most intense in the northeastern quadrant, owing to frontal convection $\mathrm{A}$; this will be discussed further in Fig. 5. Figs. $4 \mathrm{~b}$ and $4 \mathrm{f} \mathrm{dem-}$ onstrate that the frontal-convection-driven RAF results in positive VTEN (i.e., spinup process) beyond a $200-\mathrm{km}$ radius, especially in the northeastern quadrant. In other words, frontal convection increases wind speed in the outer region, generating outer local wind maximum. A strong inflow region is moving westward at the early phase of the pre-reintensification stage (Figs. 3c,g and $4 \mathrm{~g}$ ), because frontal convection $\mathrm{A}$ is advected cyclonically. In response to the westward movement of the frontal convection A (i.e., positive RAF region), the positive VTEN region propagates 


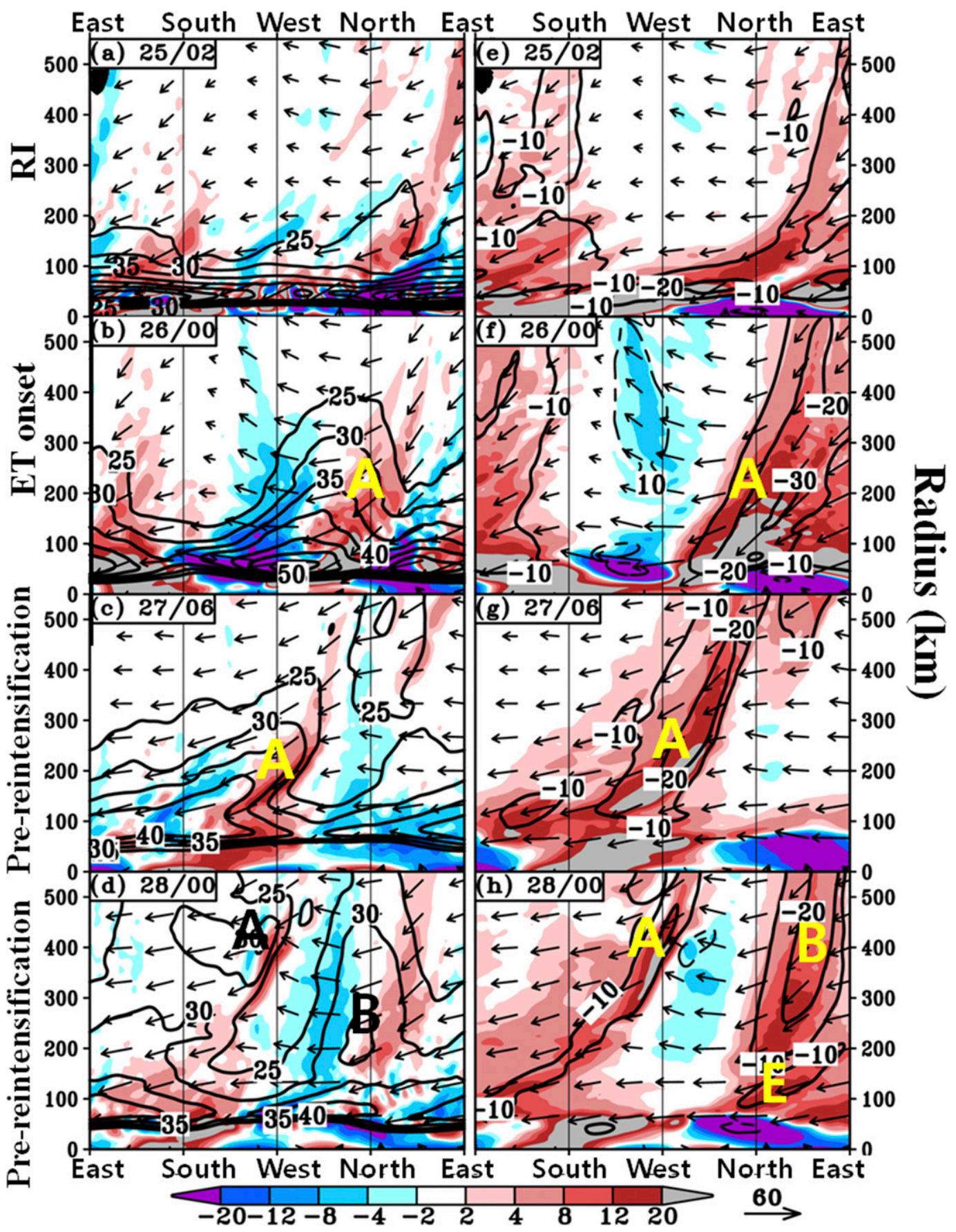

FIG. 4. (a)-(d) Radius-azimuth cross section of the quasi-Lagrangian tendency of tangential wind following the tangential flow (shaded; $\mathrm{m} \mathrm{s}^{-1} \mathrm{~h}^{-1}$ ), tangential velocity (black contours; $5 \mathrm{~m} \mathrm{~s}^{-1}$ interval above $25 \mathrm{~m} \mathrm{~s}^{-1}$ ), and horizontal (i.e., tangential and radial) flow vectors at $0.5-\mathrm{km}$ level at 25/02, 26/00, 27/06, and 28/00, respectively. Vertical axis indicates the distance $(\mathrm{km})$ from the storm center. (e)-(h) As in (a)-(d), but for radial absolute vorticity flux (RAF; shaded; $\mathrm{m} \mathrm{s}^{-1} \mathrm{~h}^{-1}$ ), and radial velocity [solid (dashed) contours; $10 \mathrm{~m} \mathrm{~s}^{-1}$ interval below $-10 \mathrm{~m} \mathrm{~s}^{-1}$ (above $10 \mathrm{~m} \mathrm{~s}^{-1}$ )]. Local wind maxima and radial inflow associated with two frontal convective regions and eyewall-like convection are denoted as A, B, and E, respectively. 


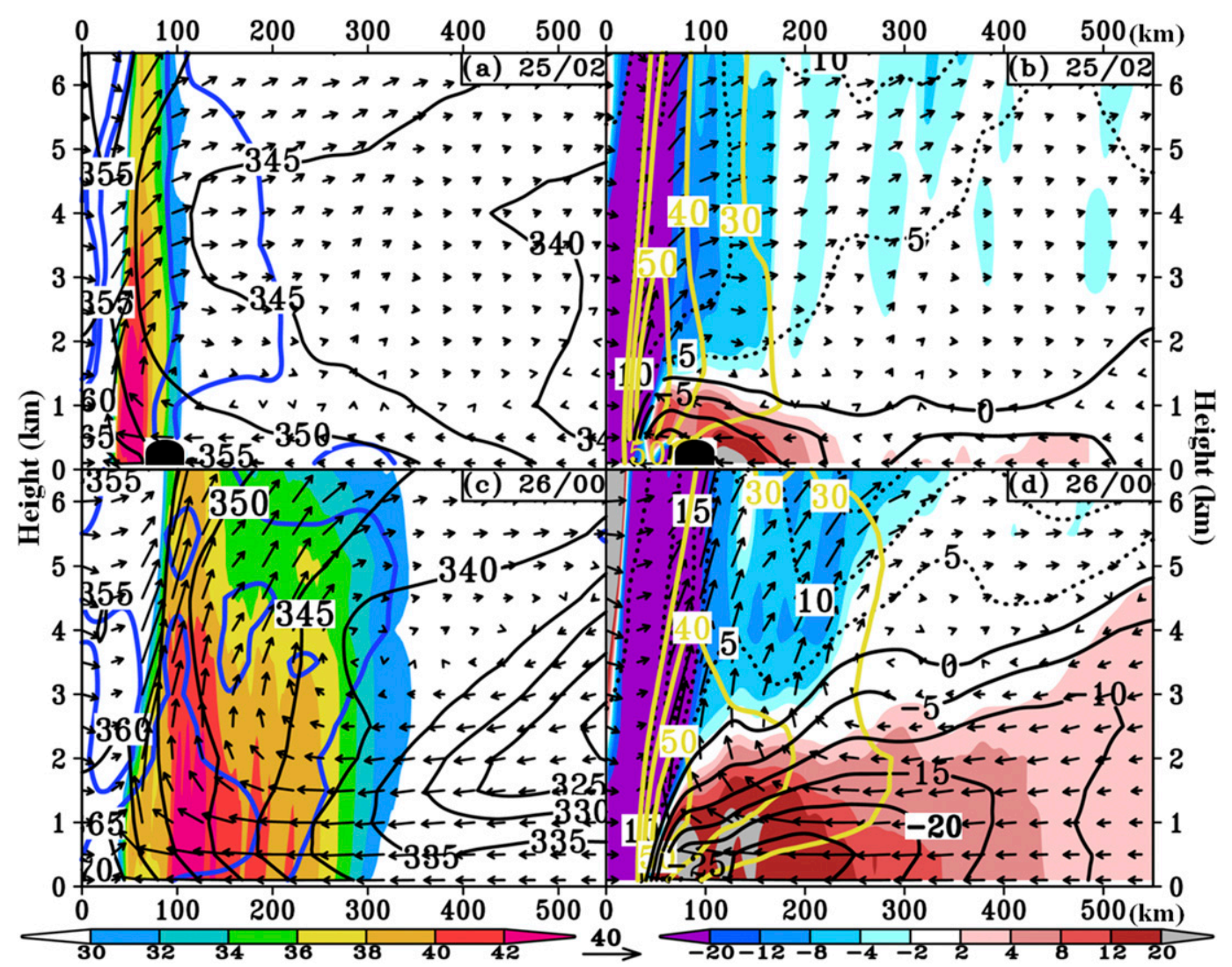

FIG. 5. Radius-height cross section of (a) radar reflectivity (shaded; $\mathrm{dB} Z$ ), equivalent potential temperature (black contours; 5-K interval), and scalar frontogenesis function [blue contours at -4 , and $-12 \mathrm{~K}\left(100 \mathrm{~km}^{-1} \mathrm{~h}^{-1}\right.$ ] at 25/02, (b) radial absolute vorticity flux (shaded; $\mathrm{m} \mathrm{s}^{-1} \mathrm{~h}^{-1}$ ), tangential velocity (yellow contours; $10 \mathrm{~m} \mathrm{~s}^{-1}$ interval above $30 \mathrm{~m} \mathrm{~s}^{-1}$ ), and radial velocity (black contours; $5 \mathrm{~m} \mathrm{~s}^{-1}$ interval; positive values are dotted, negative values are solid) at 25/02. In-plane flow vectors (vertical velocity multiplied by 30 ) are superimposed. Fields are azimuthally averaged over the segments that are indicated by two purple lines in Fig. 3. Black shading indicates terrain over Cuba. (c),(d). As in (a)and (b), but for 26/00.

cyclonically, as shown in Fig. 4c. As a result, at 0600 UTC 27 October, cyclonic tangential wind expands in the southwestern quadrant. Frontal convection A reaches west of Sandy around 0000 UTC 28 October, and positive VTEN and RAF regions are also located in the western semicircle (Figs. 3d and $4 \mathrm{~d}, \mathrm{~h})$. Because the source of the positive RAF area travels from the northeastern quadrant to the western semicircle between 0000 UTC 26 October (ET onset stage) and 0000 UTC 28 October (pre-reintensification stage), the cyclonic tangential wind area expands in most directions, as compared with 0200 UTC 25 October (cf. Figs. 4a,d). In Fig. 4h, "B" and "E" define the inflow associated with frontal convection $B$ and eyewall-like convection, respectively. The locations of $U=-10 \mathrm{~m} \mathrm{~s}^{-1}$ contours suggest that because of the eyewall-like convection and another frontogenetic forcing (B), two inflow regions develop in the northeastern quadrant.
The budget calculation illustrated in Fig. 4 confirms that because of the inward advection of AAM per unit radius in the outer frontal convective regions, cyclonic tangential velocity accelerates in those regions, expanding the cyclonic circulation of Sandy that was confined in the eyewall region before the ET began. In addition, budget calculation explains why local wind maxima exist outside of the frontal convective area. The right panels of Fig. 4 illustrate that it is the frontal convection induced-PBL inflow that causes the inward RAF, because the region of PBL inflow is almost in agreement with the positive RAF. Because of RAF, the low-level outer tangential flow of the hurricane accelerates in the inflow region (i.e., frontal convective region), which extends intense tangential wind to the downstream side of the frontal convection. As a result, a local wind maximum not only occurs within the frontal area but also extends outside of the frontal region. A similar pattern 
has also been seen in the observational study of Typhoon Sinlaku (2008) during The Observing System Research and Predictability Experiment (THORPEX) Pacific Asian Regional Campaign (Quinting et al. 2014). The wind flow pattern, frontogenesis function, and radar reflectivity at $1-$ and $1.5-\mathrm{km}$ levels, which were taken from high-resolution observation data, showed that (i) wind velocity accelerated when the flow passed the frontal convective area in the northeastern quadrant of Typhoon Sinlaku, which underwent ET, and (ii) as a result, the local wind maximum also expanded to the outer side of the frontal convection [see Figs. 4, 9a, and 11a of Quinting et al. (2014)].

A radial-vertical cross section of the northeast side of Sandy is shown in Fig. 5 to illustrate how frontogenesis-driven secondary circulation changes PBL inflow and RAF patterns, thereby affecting the spinup process of hurricanes during ET. When Sandy is located in a tropical region (i.e., 0200 UTC 25 October), deep convection exists only in the eyewall region ( $\sim 50-\mathrm{km}$ radius), and there is no significant temperature gradient outside the eyewall (Fig. 5a). The strongest PBL inflow, which exceeds $10 \mathrm{~m} \mathrm{~s}^{-1}$, is located in the vicinity of a $100-\mathrm{km}$ radius, generating intense RAF $\left(>20 \mathrm{~m} \mathrm{~s}^{-1} \mathrm{~h}^{-1}\right)$ near the eyewall convection (Fig. 5b). Vortex spinup occurs mostly near the eyewall, and this pattern is a typical feature of mature hurricanes that do not undergo ET (Yau et al. 2004; Smith et al. 2009). However, at 0000 UTC 26 October (Fig. 5c), cold, dry air (less than 340-K $\theta_{e}$ ) begins to penetrate into the low level of Sandy. In Fig. $5 \mathrm{c}$, the $-12 \mathrm{~K}(100 \mathrm{~km})^{-1} \mathrm{~h}^{-1}$ contour of the frontogenesis function and high radar reflectivity values near the $200-\mathrm{km}$ radius indicate the development of a frontal convection and updraft. In response to frontogenetic forcing at the outside of the eyewall, deep and intense inflow develops. Compared with the results in Fig. 5b, the $U=-10 \mathrm{~m} \mathrm{~s}^{-1}$ contour and the area in which the RAF exceeds $4 \mathrm{~m} \mathrm{~s}^{-1} \mathrm{~h}^{-1}$ extend much farther and higher. Tangential wind speed greater than $30 \mathrm{~m} \mathrm{~s}^{-1}$ expands as a result of the spinup process, which is illustrated in Fig. 4. A comparison of Figs. $5 \mathrm{~b}$ and $5 \mathrm{~d}$ demonstrates that the frontal convection can generate inward RAF to the outer region of the eyewall. This feature is somewhat similar to that discussed in the ET of Hurricane Bonnie by Evans and Hart (2008), who have claimed that, during ET, the inward AAM transport expands the wind field of Bonnie in the 600-700-hPa layer. In the case of Sandy, frontal convective regions outside of the eyewall play an important role in increasing low-level wind field through RAF.

\section{The spinup process during the reintensification}

SZ17 mentioned that repeated frontogenesis increases AAM in the outer region, which provides the precondition for reintensification. Therefore, this section discusses (i) the dynamical process that verifies the argument of SZ17 and (ii) how the PBL inflow driven by the enhanced eyewall-like convection causes the inward advection of AAM per unit radius, thus leading to reintensification. In particular, this section provides details about these two processes by applying the budget calculation to the model integration times 0900 and 1800 UTC 28 October and 0300 and 0600 UTC 29 October. These four times are chosen because they display the cyclonic propagation of frontal convection B (0900 and 1800 UTC 28 October), the development of the third frontal convection C (1800 UTC 28 October), and the reintensification stage (0300 and 0600 UTC 29 October). Figure 6 depicts the PBL structural evolution when the storm transitions from the pre-reintensification stage (28 October) to the reintensification stage (29 October).

At 0900 UTC 28 October (Figs. 6a,e; the middle of the pre-reintensification stage), frontal convection $\mathrm{B}$, which is located to the northeast of the storm center at 0000 UTC 28 October, is moving toward the western semicircle, in agreement with Figs. 1a and 1b. Frontal convection $B$ is advected to the western semicircle, while the new frontal convection $\mathrm{C}$ develops in the northeastern quadrant, as shown in 1800 UTC 28 October (Figs. 6b,f; the latter half of the pre-reintensification stage) and SZ17. Figure $6 f$ illustrates that two local wind maxima exist in the northern and southern semicircles associated with frontal convection $\mathrm{C}$ and $\mathrm{B}$, respectively. The latter is stronger than the former because frontal convection B is more intense than front C. During the reintensification stage, Sandy recurves northwestward, where a cold, dry continental air mass is located (Figs. 1a, 2a of SZ17). This motion enhances the horizontal $\theta_{e}$ gradient (i.e., baroclinicity) in the northwestern quadrant of the storm, and the eyewall-like convection begins to merge with the baroclinic zone (Figs. 6c,d). Although there is no significant frontogenesis in this quadrant (Fig. $6 \mathrm{~m}$ of SZ17), the flow pattern and $\theta_{e}$ distribution indicate that warm, moist air is directed toward cold, dry air in the vicinity of the eyewall-like convection. Therefore, warm, moist air could be lifted above the cold air along this baroclinic zone (i.e., strong- $\theta_{e}$-gradient region), which would facilitate the eyewall-like convection through latent heat release (i.e., buoyancy). This high $\theta_{e}$ air is the remaining low-level warm core of the storm, which provides energy for eyewall-like convection. Although VWS increases significantly (Fig. 6g), the 


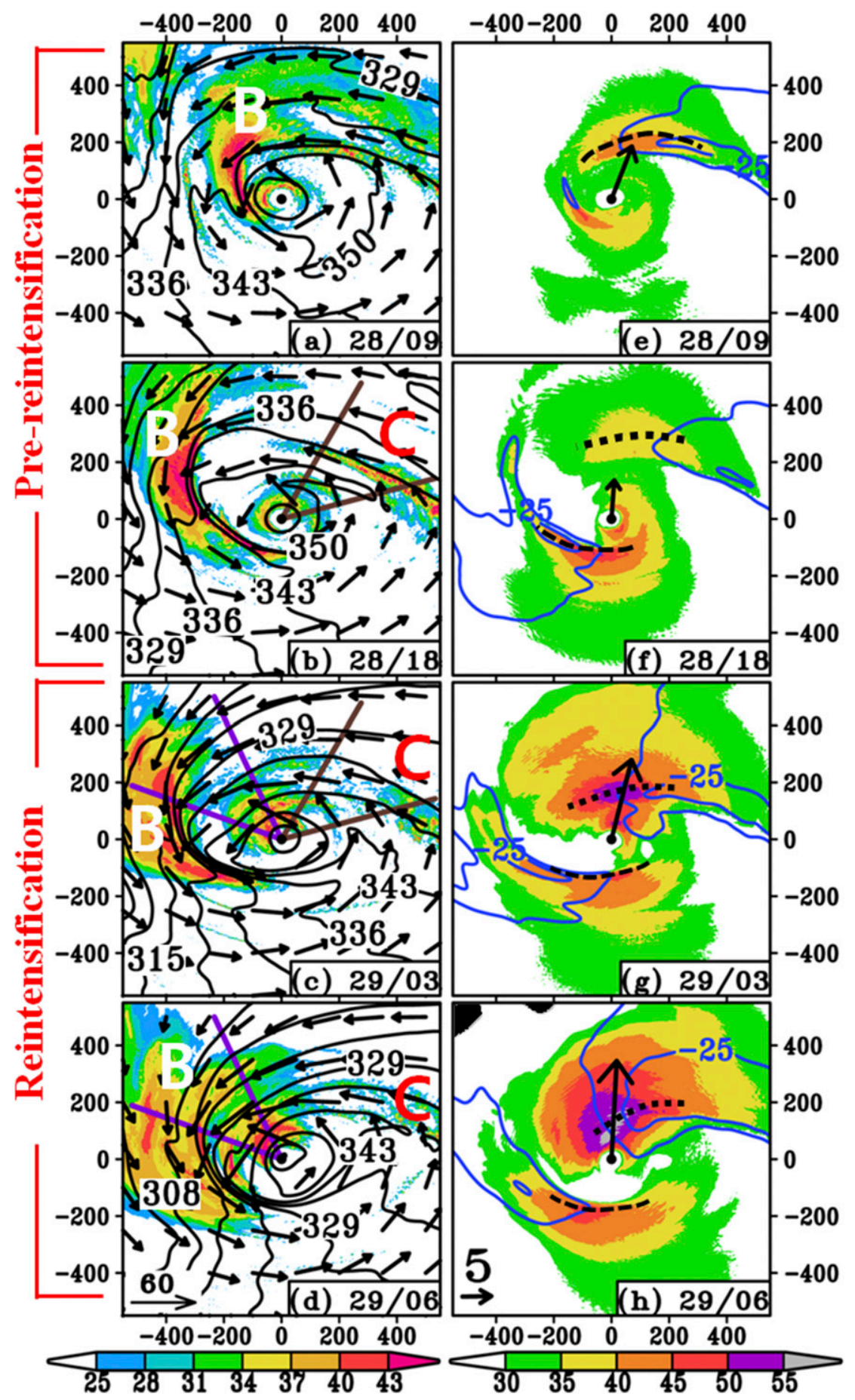

FIG. 6. As in Fig. 3, but for (top to bottom) 28/09, 28/18, 29/03, and 29/06. Frontal convective and frontogenetic regions are indicated as B and C.

eyewall-like convection is enhanced, as indicated by the increased radar reflectivity in Fig. 6c.

Reintensification begins around 0300 UTC 29 October; in Fig. $6 \mathrm{~g}$, the areal size of wind speed $40 \mathrm{~m} \mathrm{~s}^{-1}$ expands dramatically, and the maximum wind speed of the local wind maximum exceeds $50 \mathrm{~m} \mathrm{~s}^{-1}$ in the northern semicircle, whereas a local wind maximum associated with frontal convection B does not intensify. A comparison of the dotted line locations in Figs. $6 \mathrm{f}$ and $6 \mathrm{~g}$ also indicates the contraction of the local wind maximum of frontal 
convection $\mathrm{C}$ between the pre-reintensification stage and the reintensification stage. In the northeastern quadrant, Fig. $6 \mathrm{~g}$ shows that (i) the maximum of the PBL inflow develops at more than $25 \mathrm{~m} \mathrm{~s}^{-1}$, and (ii) a strong PBL inflow that exceeds $15 \mathrm{~m} \mathrm{~s}^{-1}$ penetrates within a $200-\mathrm{km}$ radius. This intense PBL inflow is driven by the strong eyewall-like convection, and one can intuitively assume that this inflow increases the inward RAF, which spins up the core region. The location of strong PBL inflow suggests that the intense local wind maximum in the northern semicircle is associated with the eyewalllike convection and frontal convection C. This reintensification continues at 0600 UTC 29 October (Fig. 6h) as the more intense PBL inflow and local wind maximum continue to increase in the northern semicircle.

Figure 7 illustrates how the PBL inflow and RAF patterns change when the storm transitions to the reintensification stage. Figures $7 \mathrm{a}$ and $7 \mathrm{~b}$ show that frontal convection B is advected to the western semicircle between 0900 and 1800 UTC 28 October, which generates another intense inflow in the southwestern quadrant at 1800 UTC 28 October, as shown in Fig. 7f. Near the $120-\mathrm{km}$ radius, the maximum of the tangential wind or RMW exists, owing to the RAF, which exceeds $20 \mathrm{~m} \mathrm{~s}^{-1} \mathrm{~h}^{-1}$ (Figs. 7b,f). Simultaneously, another band of the local wind maximum (denoted " $\mathrm{C}$ " in Fig. 7b) is located $\sim 280 \mathrm{~km}$ north of the storm center. Figure $7 \mathrm{f}$ depicts that the $U=-10 \mathrm{~m} \mathrm{~s}^{-1}$ contour and the positive RAF region in the northeastern quadrant are associated with frontal convection $\mathrm{C}$, thus indicating that the local wind maximum in this area is due to frontal convection C. Therefore, there are two local RMWs (i.e., local wind maxima) associated with two frontal convective regions at 1800 UTC 28 October. When Sandy undergoes reintensification at 0300 UTC 29 October (Fig. 7c), the RMW associated with frontal convection $\mathrm{C}$ contracts to a $150-\mathrm{km}$ radius, while the other RMW generated by frontal convection $\mathrm{B}$ does not. In addition, the maximum tangential velocity of frontal convection $\mathrm{C}$ increases to more than $50 \mathrm{~m} \mathrm{~s}^{-1}$, and VTEN in the northeastern quadrant exceeds $20 \mathrm{~m} \mathrm{~s}^{-1} \mathrm{~h}^{-1}$. The PBL flow pattern indicates that a strong PBL inflow area (exceeding $10 \mathrm{~m} \mathrm{~s}^{-1}$ ) stretches to around a $50-\mathrm{km}$ radius, and the inflow in the core region is enhanced (Fig. 7g). This inflow pattern increases the transport of high-momentum air into the storm's core region, thus accelerating the tangential wind velocity (i.e., intensifying the wind in the northern semicircle). As already discussed in Figs. 6c and $6 \mathrm{~g}$, this intense inflow appears to be generated by the strong eyewall-like convection. At 0600 UTC 29 October (Fig. 7d), the northern local wind maximum further intensifies to $\sim 55 \mathrm{~m} \mathrm{~s}^{-1}$ as the RMW contracts to $\sim 100 \mathrm{~km}$. Moreover, Fig. $7 \mathrm{~h}$ illustrates that a very strong PBL inflow, marked as the $U=-20 \mathrm{~m} \mathrm{~s}^{-1}$ contour, exists near the $100-\mathrm{km}$ radius. In response to the development of the inflow (i.e., enhanced RAF), a positive VTEN of greater than $20 \mathrm{~m} \mathrm{~s}^{-1} \mathrm{~h}^{-1}$ occupies most of the core region in the northeastern quadrant. That is, the region where the acceleration of the tangential flow occurs is large in this quadrant.

The distribution of tangential wind in Figs. $4 a-d$ and $7 \mathrm{a}-\mathrm{d}$ shows how continuous frontogenesis during the pre-reintensification stage increases AAM in the outer region (i.e., beyond a $200-\mathrm{km}$ radius) and provides the precondition for reintensification. Where frontal convection exists, there is an inflow that blows toward this outer convective region, causing inward RAF. Frontal convection A, which develops around 26 October or the ET onset stage, initiates the wind field expansion via RAF (Figs. 4b,f), and during the pre-reintensification stage (27 and 28 October) the subsequent frontogenesis (i.e., frontal convection B and C) outside of the 200-km radius continues to generate $\mathrm{RAF}$, which increases (i.e., spinning up) the tangential wind speed in the outer region. As a result, the air beyond the $200-\mathrm{km}$ radius contains high AAM, but, as shown in Figs. $4 \mathrm{~h}$ and $7 \mathrm{f}$, the outer frontal convection does not induce enough strong inflow to increase the wind speed in the core. Moreover, although frontal convection spins up the outer region, it cannot substantially enhance the core region wind, which is directly related to storm intensity. If a strong PBL inflow that extends from the outer region to the core region develops, the inflow will transport highAAM air from the outer region to the core region and generate an intense local wind maximum within a $200-\mathrm{km}$ radius (Figs. $7 \mathrm{~g}, \mathrm{~h}$ ). In addition, when this happens, the core of the local wind maximum (i.e., RMW) contracts. SZ17 noted that the contraction of the storm vortex on 29 October is associated with reintensification (Fig. 7a of SZ17), in agreement with findings from Galarneau et al. (2013). This study shows that the contraction of RMW occurs in the northern semicircle, where an intense local wind maximum is generated. The magnitude of the PBL inflow in the northern semicircle is also important, because stronger PBL inflow results in more inward AAM advection. This explains why Sandy reintensifies on 29 October, at which point the PBL inflow reaches its maximum intensity.

For analysis of the vertical structure of the inflow and RAF, when the storm is moving into the reintensification stage, the radial-height cross sections of the inflow region at 1800 UTC 28 October and 0300 UTC 29 October are compared in Figs. 8a-d. At 1800 UTC 28 October (Figs. 8a,b), the PBL inflow is weak in the core region and mostly exists beyond the frontal convection $\mathrm{C}$ 


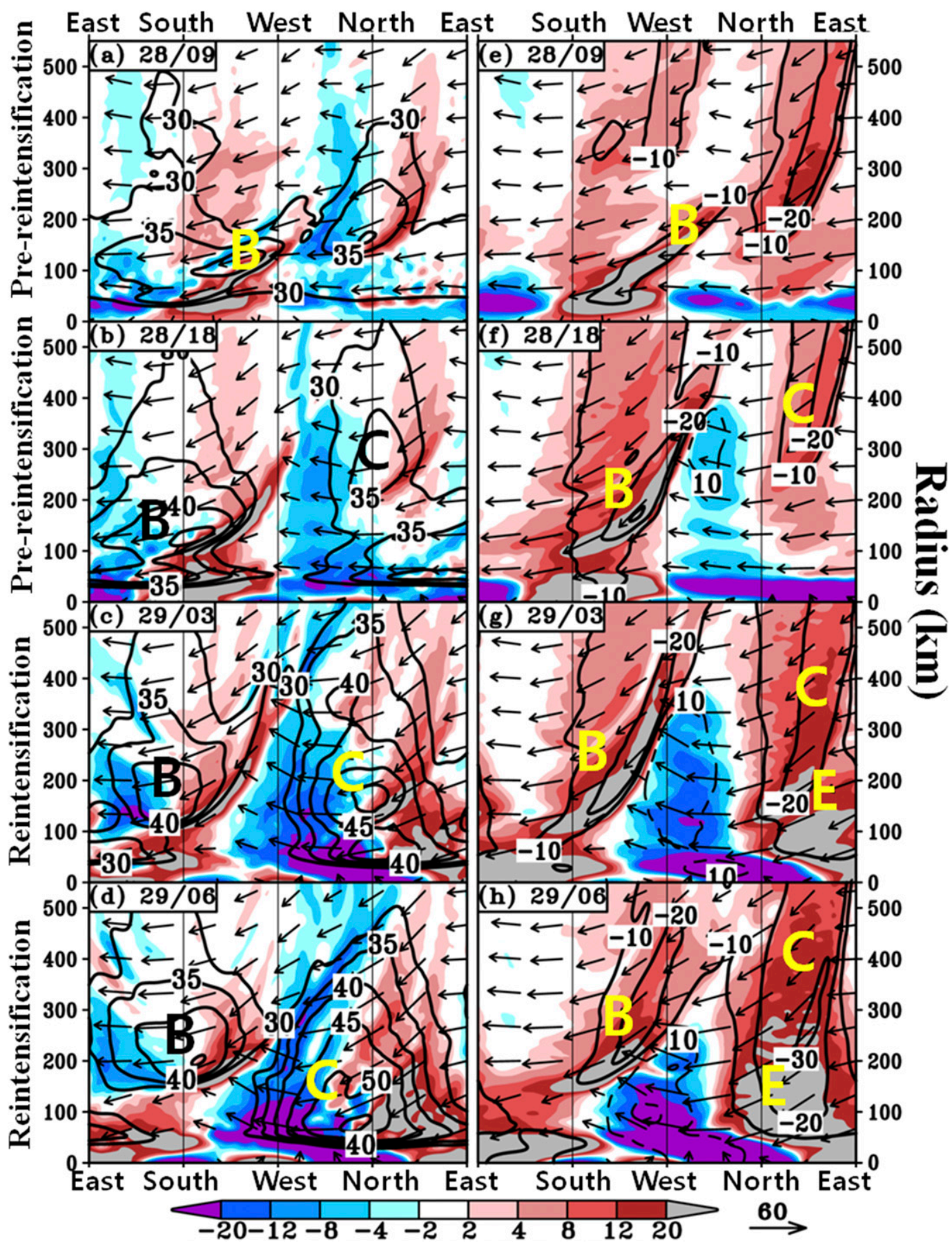

FIG. 7. As in Fig. 4, but for (top to bottom) 28/09, 28/18, 29/03, and 29/06. (left) Tangential velocity is contoured at a $5 \mathrm{~m} \mathrm{~s}^{-1}$ interval, starting at $30 \mathrm{~m} \mathrm{~s}^{-1}$. Local wind maxima and radial inflow associated with two frontal convective regions and eyewall-like convection are denoted as B, C, and E, respectively.

( $\sim 250-\mathrm{km}$ radius), as indicated by the areal size of the $U=-10 \mathrm{~m} \mathrm{~s}^{-1}$ contour and the maximum value of $U$. Figure $8 \mathrm{~b}$ also shows that the RAF in the PBL is mostly less than $8 \mathrm{~m} \mathrm{~s}^{-1} \mathrm{~h}^{-1}$. However, at 0300 UTC 29 October (Figs. 8c,d), the inflow below the 2-km level is enhanced in response to the development of eyewall-like convection. A strong convective signal is not shown in Fig. 8c, because the eyewall-like convection is not located in this section. As deep PBL inflow intensifies, RAF reaches almost $20 \mathrm{~m} \mathrm{~s}^{-1} \mathrm{~h}^{-1}$ in the core region, and the storm vortex is 


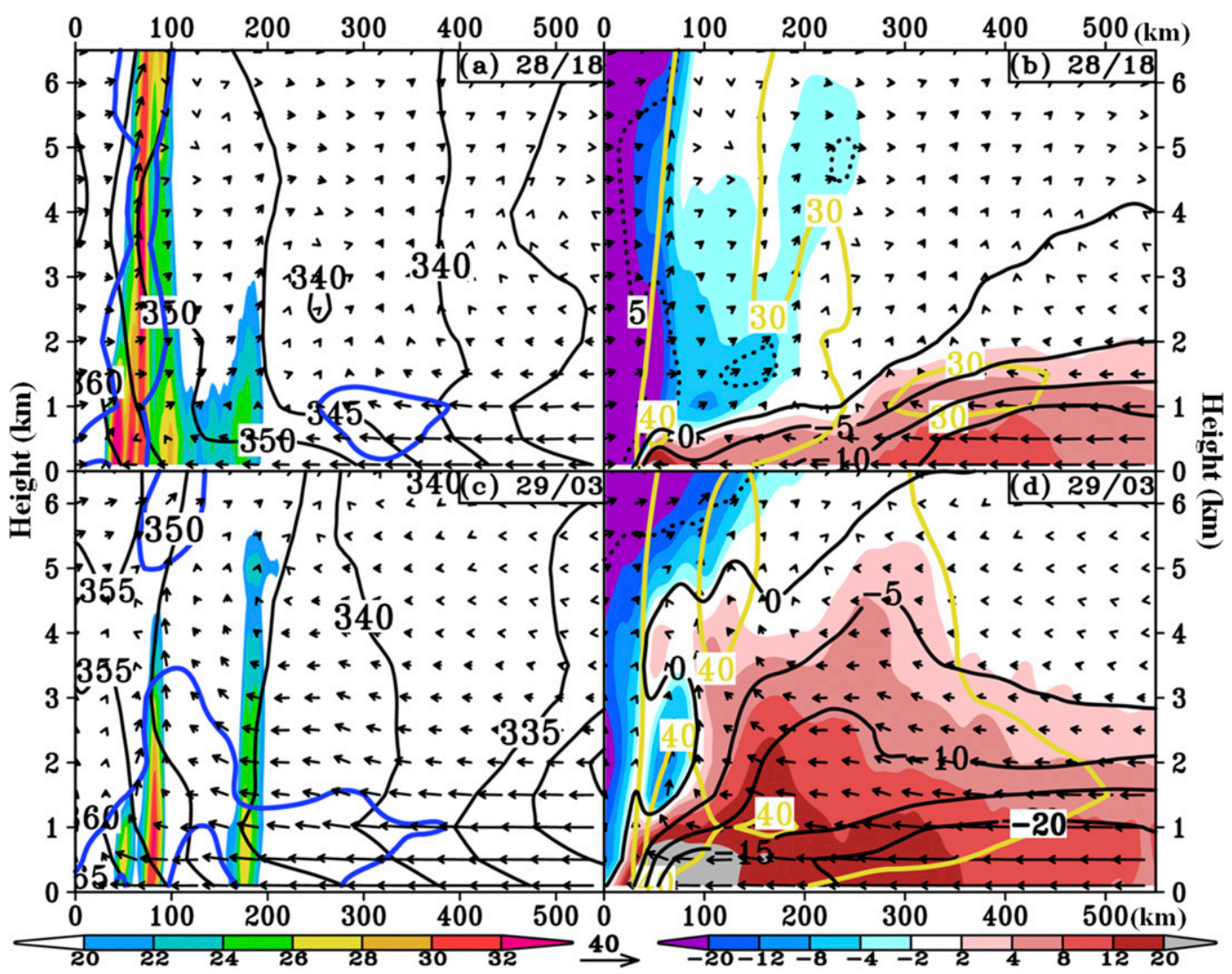

FIG. 8. As in Fig. 5, but for (a),(b) 28/18 and (c),(d) 29/03. The scale of radar reflectivity (shaded; dBZ) is different than in Fig. 5 and fields are azimuthally averaged over the segments that are indicated by two brown lines in Figs. $6 \mathrm{~b}$ and $6 \mathrm{c}$.

intensified, as indicated by the expansion of the $V=$ $30 \mathrm{~m} \mathrm{~s}^{-1}$ contour. Interestingly, the areal sizes of the inflow and positive RAF region are much larger than those when Sandy does not undergo ET (cf. Figs. 5b and $8 \mathrm{~d}$ ).

\section{The relation between the evolution of eyewall-like convection and reintensification}

It is claimed in section 5 that inflow in the northeastern quadrant is intensified by the eyewall-like convection on 29 October, which ultimately reintensifies Sandy. Thus, the evolution of eyewall-like convection is important to understand the fluctuations in storm intensity. Because Sandy is mostly under strong VWS in the midlatitudes (SZ17), the time-azimuth cross section of radar reflectivity is plotted to trace the movement of convection in the eyewall-like structure (Fig. 9a). Sandy has a large core region, and the convection associated with the eyewall-like structure is scattered roughly between a 50- and 200-km radius (Figs. 3 and 6); therefore, the radar reflectivity field in Fig. 9a is averaged from a $100-$ to $150-\mathrm{km}$ radius to obtain an approximate and representative feature. Because section 5 shows that the most intense inflow $\left(>20 \mathrm{~m} \mathrm{~s}^{-1}\right)$ usually occurs in the northeastern quadrant and implies that variation in this quadrant inflow is closely related to eyewall-like convection, Fig. 9b displays the PBL radial velocity, which is averaged in the northeastern quadrant. Figure $9 \mathrm{~b}$ displays the $1-5-\mathrm{km}$ layer-averaged value of core region's vertical mass flux $[\Sigma(\rho w D)]$, the summation of $\rho w D$ (where $\rho, w$, and $D$ are density, vertical velocity, and gridcell area, respectively) of each grid points in the core region. Only grid points within a $\sim 200-\mathrm{km}$ radius are included in $\Sigma(\rho w D)$. Vertical mass flux and PBL inflow are used to estimate the intensity of secondary circulation driven by the eyewall-like convection that emerges after 27 October; stronger vertical mass flux (i.e., convective activity) leads to stronger inflow.

Figure 9a clearly shows that during the cyclonic propagation pattern of eyewall-like convection, especially when VWS exceeds $10 \mathrm{~m} \mathrm{~s}^{-1}$, the convection is initiated (dissipated) in the downshear right (upshear left) side, and this pattern is similar to that observed in other hurricanes (Black et al. 2002; Reasor et al. 2009). Between 0600 UTC 27 October and 0900 UTC 28 October, 


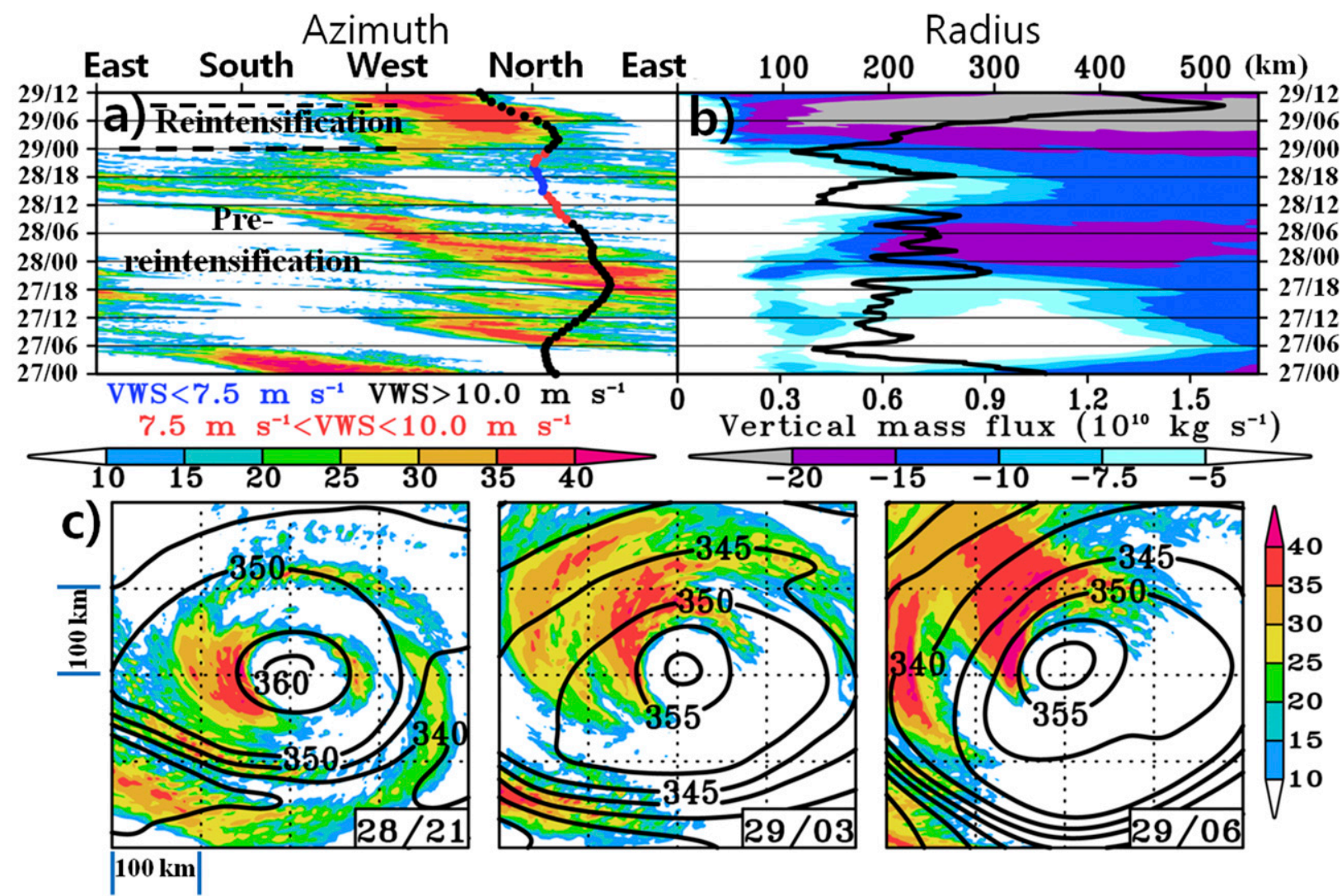

FIG. 9. Time-azimuth cross section of (a) 3-km radar reflectivity (shaded; dBZ) averaged over a 100-150-km radius and the direction and magnitude of 200-850-hPa VWS. (b) Time-radius cross section of $0.5-\mathrm{km}$ radial velocity (shaded; $\mathrm{m} \mathrm{s}^{-1}$ ) averaged over northeastern quadrant, and the time series of a 1-5-km layer-averaged vertical mass flux (black line; $10^{10} \mathrm{~kg} \mathrm{~s}^{-1}$; the scale is given in the lower axis) within a $200-\mathrm{km}$ radius. In (a) and (b), fields are not temporally averaged to obtain instantaneous features. and the time window is 27/00-29/12. (c) The 3-km radar reflectivity (shaded; dBZ), and 1-km equivalent potential temperature (contours; 5-K interval) at different time steps (given in the lower-right corners). Each box indicates a $100 \mathrm{~km} \times 100 \mathrm{~km}$ area, and the hurricane center is located at the center of the domain.

the vertical mass flux and northeast quadrant inflow ${ }^{6}$ fluctuate but generally increase during this period (Figs. 9a,b). Between 0900 and 2100 UTC 28 October, as VWS relaxes to $6.2 \mathrm{~m} \mathrm{~s}^{-1}$, the eyewall-like convection is able to propagate to the southern semicircle or upshear side, and it is often located in the upshear side during this period. Although the vertical mass flux in this period spikes to $\sim 0.8 \times 10^{10} \mathrm{~kg} \mathrm{~s}^{-1}$ temporally at 1800 UTC 28 October, it is generally lower

\footnotetext{
${ }^{6}$ Because the simulated frontal convection region B temporarily exists closer to the center, as compared with observations around 0600 UTC 28 October (Figs. 1a,b), the simulated PBL inflow associated with this frontal convection may have penetrated farther into the core region than the observed PBL inflow. However, the appendix and section 2 explain that, despite this temporary discrepancy between the simulation and observations, the simulation still reproduces the evolution of observed wind and the convective structure well during the following time periods.
}

than that in the previous phase (1200 UTC 27 October-0900 UTC 28 October), thus indicating relatively lower convective activity in this period (Fig. 9b). In response to the weakening of convective activity, the inflow intensity also decreases, thus implying weaker secondary circulation than that in the previous period.

However, there is a significant intensification of core region convection and inflow during the reintensification stage. Figure 9 illustrates that just after 2100 UTC 28 October, the VWS begins to increase rapidly while the convective areas are advected from the east to north of the storm center. A comparison between 2100 UTC 28 October and 0300 UTC 29 October of Fig. 9c also shows that the radar reflectivity increases in the $50-200-\mathrm{km}$ annulus of the northwestern quadrant. As indicated by high radar reflectivity $(>25 \mathrm{dBZ})$ in Fig. 9a, convection is anchored in the northwestern quadrant and increases rapidly after 0000 UTC 29 October. As shown in the 
comparison between 2100 UTC 28 October and 0300 UTC 29 October of Fig. 9c, the reasons for these changes are that (i) after 0000 UTC 29 October, convective cells, originally located east of the storm center, merge with the baroclinic zone as they move into the northwestern quadrant, and (ii) the increase in baroclinicity (i.e., $\theta_{e}$ gradient) facilitates the development of convection. As baroclinicity increases in this quadrant at 0600 UTC 29 October, the eyewall-like convection is anchored in this region, and, as a result, the cyclonic propagation of convection ceases (Figs. 9a,c). In response to the eyewall-like convection development, the secondary circulation of the storm intensifies vigorously; between 0000 and 0600 UTC 29 October, the vertical mass flux increases from $0.4 \times 10^{10}$ to $0.96 \times 10^{10} \mathrm{~kg} \mathrm{~s}^{-1}$, and the PBL inflow consequently reaches a maximum on 29 October (Fig. 9b).

In Fig. 9, the convective activity in the core region is enhanced when the eyewall-like convection is located in the downshear side during the periods 0600 UTC 27 October-0900 UTC 28 October and 0000-0900 UTC 29 October. However, the activity of eyewall-like convection is much stronger in 0000-0900 UTC 29 October when the eyewall-like convection significantly merges with the baroclinic zone, thus suggesting that the merging process between the eyewall-like convection and baroclinic zone plays a more important role in increasing inner core convection (and reintensification) than the forcing of VWS in the downshear side.

Figure 10 shows that as the eyewall-like convection merges with the baroclinic zone during the reintensification stage, the eyewall-like convection structure exhibits a deep slantwise convection (updraft) feature near the $200-\mathrm{km}$ radius. The eyewall-like convection appears to have a deep slantwise convection pattern because the warm, moist air associated with low-level warm core is lifted by the cold, dry air, as discussed in section 5. In particular, increasing of updraft within a $300-\mathrm{km}$ radius shows the enhancement of eyewalllike convection, which leads to the development of intense inflow in the northeastern quadrant, as shown in Fig. 9b.

Evans et al. (2017) have noted that the energy of Sandy comes from tropical (i.e., latent heat release) and extratropical sources (i.e., horizontal temperature gradient), on the basis of Galarneau et al. (2013) and SZ17. This study clearly confirms this idea, because the eyewall-like convection is also enhanced when baroclinicity in the core region increases, as shown in Figs. 6 and 9. The horizontal temperature gradient and the deep eyewall-like convection are two important factors that affect a storm's intensity. Although the eyewall

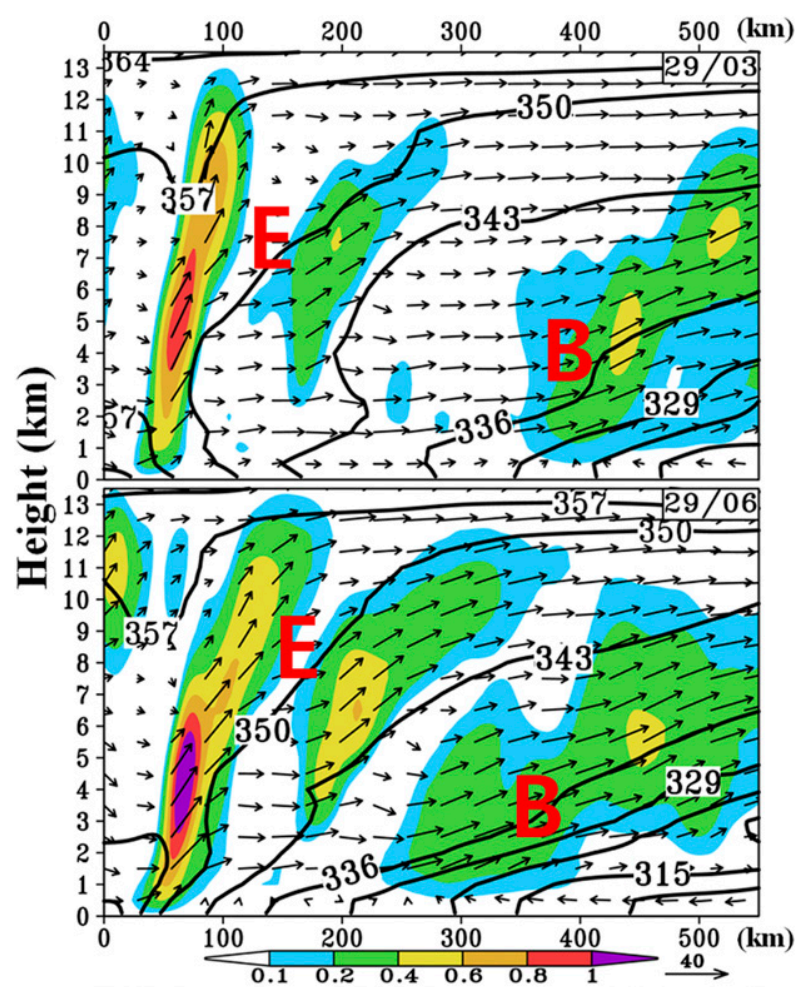

FIG. 10. Radius-height cross section of vertical velocity (shaded; $\mathrm{m} \mathrm{s}^{-1}$ ), equivalent potential temperature (black contours; 7-K interval), and in-plane flow vectors (vertical velocity multiplied by 30 ) at (top) $29 / 03$ and (bottom) $29 / 06$. Fields are azimuthally averaged between two purple lines denoted in Figs. $6 \mathrm{c}$ and $6 \mathrm{~d}$. Frontal convection and eyewall-like convection are denoted as B and E, respectively.

appears to be eroded during ET (Klein et al. 2000), Sandy, and a few other hurricanes that intensified during the transformation stage of ET, maintains the eyewall-like convection during the reintensification period, thus supporting that eyewall-like convection is still important in the reintensification process of ET events (Abraham et al. 2004; Evans and Prater-Mayes 2004; Nguyen and Molinari 2012; Blake et al. 2013). During the reconnaissance mission of the reintensifying Hurricane Michael (2000) and Sandy (2012), reintensification ended when the eyewall-like convection dissipated or weakened (Abraham et al. 2004; Blake et al. 2013). During the ET of Irene (1999), the hurricane underwent RI when the radar reflectivity of the eyewall-like convection increased (Nguyen and Molinari 2012). Therefore, (i) Sandy would not reintensify if the eyewall-like convection dissipates earlier, before the storm enters into the deep midlatitudes, and (ii) the activity and the presence of the eyewall-like convection during ET may be a useful indicator that estimates the reintensification for transforming hurricanes. 


\section{Summary}

This study examines the structural and intensity changes of Sandy from a vortex spinup perspective by utilizing the quasi-Lagrangian azimuthal momentum equation and $1.667-\mathrm{km}$-resolution numerical simulation data. This study is unique in that it examines (i) the detailed dynamic process associated with the outer frontal convection that expands the wind field of a hurricane in the ET event and (ii) the importance of eyewall-like convection on the reintensification of hurricanes during the transformation stage in ET.

As illustrated by the schematic in Fig. 11, before ET begins, the local maximum wind is located only in the eyewall region (Fig. 11a; RI stage). In the ET onset stage (Fig. 11b), the local wind maximum and the cyclonic flow of Sandy begin to expand because frontal convection A develops to the northeast of Sandy. However, on 27 October, when the storm evolves into the prereintensification stage, as Sandy moves northeastward, and frontal convection A (i.e., baroclinic zone) propagates away from the core region, an inner eyewall-like convection emerges. Consequently, after 27 October, during the pre-reintensification stage (Fig. 11c), Sandy has an eyewall-like structure and warm-core characteristics similar to those of tropical cyclones, but it also possesses a frontal structure in the outer region, similarly to extratropical cyclones. Because the baroclinic zone is located in the outer region of the storm for nearly 2-3 days (i.e., 27-28 October), the long-lasting confluence between Sandy's warmer cyclonic circulation and the colder easterly flow results in repeated frontogenesis in the northeastern quadrant (Fig. 11c). Quantitative analysis verifies that frontal convective regions result in local wind maxima in the outer region of Sandy (i.e., expanding the storm's wind field) via the inward RAF, because the PBL inflow that blows toward frontal convective regions develops in the outer region. Cyclonic tangential flow of the outer region accelerates at locations where PBL radial inflow occurs in the frontal convective region, because this front-driven inflow causes inward RAF. As a result, as illustrated in Fig. 11c, local wind maxima associated with frontal convection not only develop within the front region but also extend on the downstream side (i.e., outside) of the frontal convection. Therefore, local wind maxima can even exist where strong convection does not occur. However, although frontal convective regions expand and enhance the outer wind field of Sandy, frontal convection does not cause the intensification alone. During the reintensification stage (Fig. 11d), intense inflow, which extends from the outer region to the core region, develops in the northeastern quadrant.
This strong inflow acts as a channel between the outer and core regions; it increases the transportation of local-wind-maximum-driven high-momentum air (i.e., inward RAF), from the outer region to the core region and spins up the cyclonic tangential wind near the center of Sandy.

This research verifies that, whereas frontal convective regions enhance only the outer wind field, the eyewall-like convection increases the core region wind speed and ultimately the storm intensity. The development of the intense radial inflow is directly related to the activity of large eyewall-like convection at the core region of the storm. And the activity of eyewall-like convection is influenced by the baroclinic zone. When the baroclinic zone, in the northwestern section of the storm, approaches the storm center where a warm core exists (owing to the storm's poleward movement), it enhances the eyewall-like convection because warm, moist air in the core region is lifted by the cold, dry air associated with the baroclinic zone. In response to the enhancement of eyewall-like convection, intensified inflow occurs in the northeastern quadrant and increases the inward transportation of high-momentum air associated with the outer local wind maximum, thus enhancing the spinup process of the core region. The PBL inflow reaches its maximum intensity on 29 October, thereby explaining why the storm reintensifies before landfall.

These results suggest that during ET, (i) if eyewalllike convection is maintained and (ii) is invigorated by external forcing (e.g., baroclinicity) that enhances ascending motion, hurricanes could reintensify. Warm core and deep eyewall-like convection of the core region determine the storm intensity, whereas baroclinicity results in a local wind maximum in the outer frontal convective regions and modulates the activity of the eyewall-like convection. This eyewall-like feature has also been observed in other reintensifying hurricanes in the transformation stage of ET, thus indicating that the enhancement of eyewall-like convection's activity may be an important indicator that determines the intensification of hurricanes during ET. However, further study of other factors regarding reintensification may be required, because, as illustrated in Fig. 10 of SZ17, the upper-level trough is located in the vicinity of Sandy on 29 October. Previous studies (Thorncroft and Jones 2000; Klein et al. 2002) have shown that the upper-level trough and jet stream contribute to the reintensification of hurricanes that complete ET. The upper-level synoptic condition appears to also affect the reintensification of Sandy. This issue will be studied further by using 


\section{a) Rapid intensification stage}

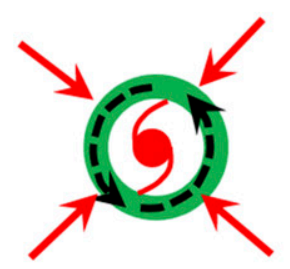

b) ET onset \& early phase of prereintensification stage

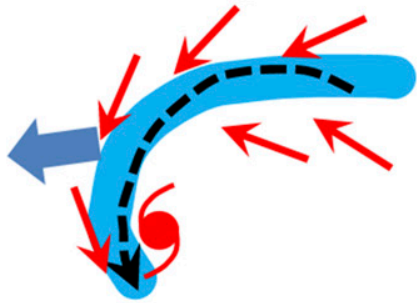

Development and cyclonic propagation of frontal convection (outer wind maximum)

\section{c) Pre-reintensification stage}

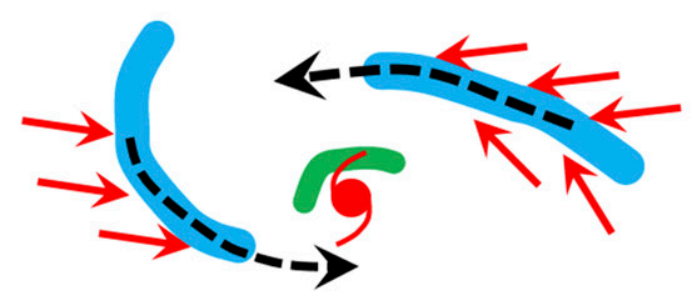

Repeated frontogenesis in the northeastern quadrant. Two outer frontal convective regions and inner eyewall-like convection

\section{d) Reintensification stage}

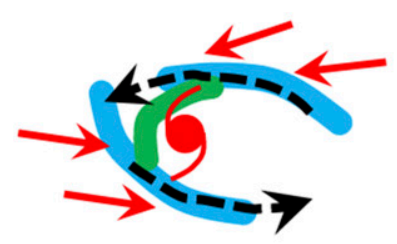

Contraction of outer wind maximum and merging between baroclinic zone and eyewall-like convection
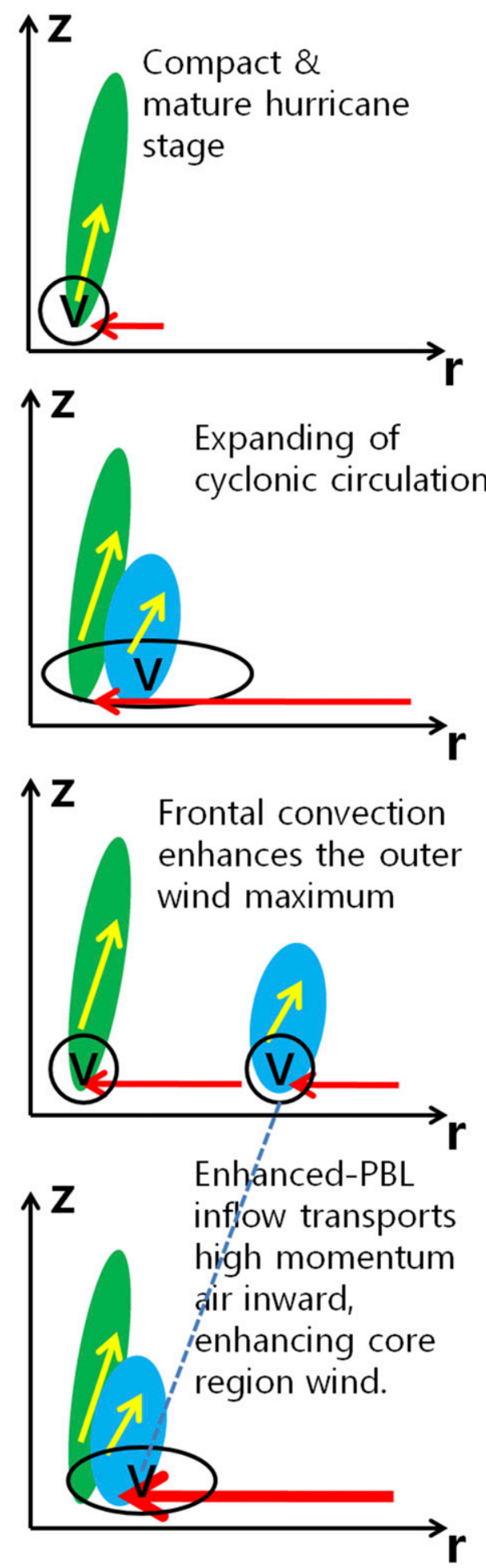

FIG. 11. Schematic of the evolution of Sandy that summarizes the evolution of Sandy in a (left) horizontal view and (right) radius-height cross section. The radius-height cross section is in the northern or north-northeastern part of Sandy roughly. (a) First intensity peak as a mature hurricane on 25 Oct. (b) Onset of extratropical transition on 26-27 Oct. (c) Pre-reintensification on 27-28 Oct. (d) Reintensification on 29 Oct. Here, green shading, blue shading, black dotted arrows, red solid arrows, and hurricane symbol indicate eyewall or eyewall-like convection, frontal convection, local wind maxima, PBL inflow that blows toward the convection, and the storm center, respectively. In the right panels, the horizontal axis, "V," and the yellow arrows indicate radial direction from the center, local wind maxima, and updraft associated with convection, respectively. 


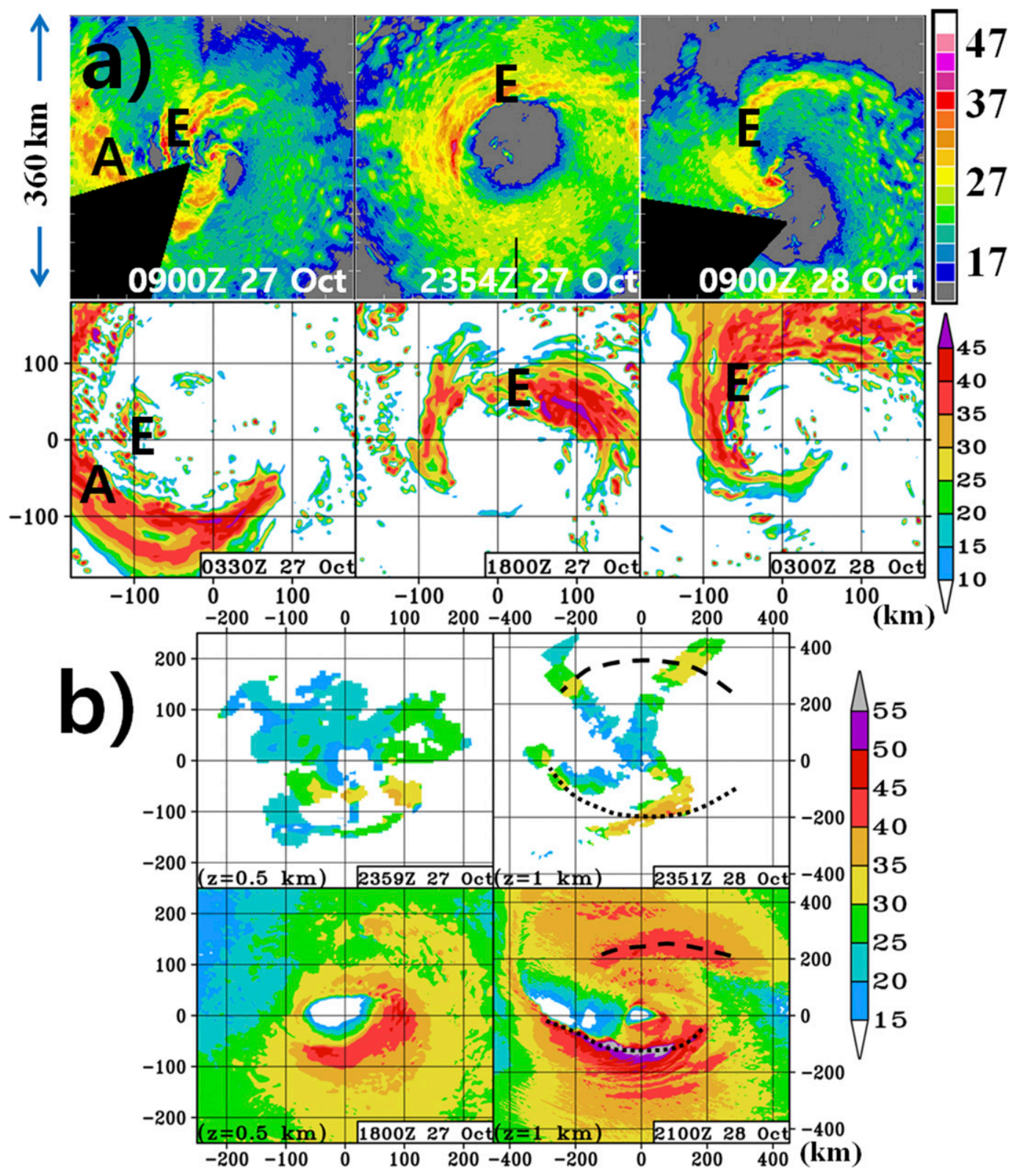

FIG. A1. (a) Comparison of radar reflectivity near 3.5-km level (shaded; $\mathrm{dB} Z$ ) from (top) observations and (bottom) WRF simulation. Frontal convection and eyewall-like convection are denoted as A and E, respectively. (b) As in (a), but for low-level wind speed (shaded; $\mathrm{m} \mathrm{s}^{-1}$ ); vertical level ( $\mathrm{km}$ ) is given in the lower-left corner. Horizontal and vertical axes show the distance $(\mathrm{km})$ from the storm center in which both observations and simulations display similar areal size. Simulated fields are not temporally averaged. Observational data and color label for observed radar reflectivity are obtained from the Hurricane Research Division web page.

a series of sensitivity experiments or the same model simulation.

Acknowledgments. The WRF Model integration was completed on the Discover machine at the NASA Center for Climate Simulation during the author's Ph.D. study at the University of Maryland (UM).
The author would like to express appreciation to three reviewers who helped substantially improve this manuscript. The author is also grateful for William Miller (UM) and Daniel Nielson (FNMOC) for discussions and editorial assistance. The author thanks Jeff Henrikson (UM) and KyoungSoo Lee for computational support and financial support, respectively. The 
GridSat-B1 satellite data are developed and provided by Knapp et al. (2011).

\section{APPENDIX}

\section{Storm Structure from Simulation and Observations}

Figure A1 displays the simulated and observed features of Sandy during the pre-reintensification stage. It should be noted that after 0000 UTC 27 October, the simulated Sandy follows the observed track but moves a bit faster than the real Sandy (Fig. 1d). Therefore, comparison of an observed storm against the simulation, on the basis of the time at which the simulated storm undergoes the observed structural or intensity changes, would be meaningful. At 0900 UTC 27 October, the observed frontal convection A was still attached to the eyewall-like convection but was about to become detached. This pattern is simulated at 0330 UTC 27 October (Fig. A1), which is $2.5 \mathrm{~h}$ earlier than 0600 UTC 27 October, when two simulated convective regions are already separated (Fig. 3c). The observed wind structure in the core region around 0000 UTC 28 October (Fig. A1b; $\sim 6 \mathrm{~h}$ before the development of frontal convection $\mathrm{B}$ ) resembles the simulated structure of 1800 UTC 27 October more than that of 0000 UTC 28 October (Fig. 3h), because the strongest wind exists primarily in the southeastern quadrant. The observed sheared convective structure at 0000 UTC 28 October resembles the simulation at 1800 UTC 27 October (Fig. A1a). The observed arc-shaped convection or eyewall-like convection that encircles the northwest of the storm center at 0900 UTC 28 October (several hours after the development of frontal convection B) is reproduced in the simulation at $0300 \mathrm{UTC}$ 28 October, although the simulated frontal convection B is located northeast of this convection. The observed wind field around 0000 UTC 29 October, when the storm is about to transition to reintensification from the pre-reintensification stage, reveals that a relatively stronger (weaker) local wind maximum existed in the southern (northern) semicircle (dotted lines in Fig. A1b). These two wind maxima are also reproduced well by the simulation at 2100 UTC 28 October, and the simulated northern wind maximum intensifies during the reintensification stage, similarly to the observations (cf. Fig. 6n of SZ17 and Fig. 1c herein). These results verify that the temporary discrepancy between the simulation and observation around 0600 UTC 28 October, discussed in section 2, does not influence the evolution of the storm during the ensuing period. Additionally, Fig. 5 of SZ17 supports that the simulation well reproduces two frontal convective regions and eyewall-like convection after 0600 UTC 28 October.

Figure A1 verifies that what occurred during the real Sandy occurs during similar time frames in the WRF simulation, thus supporting that the simulation reproduces the evolution of the observed storm well. Because of the faster movement of the simulated Sandy, after 27 October, the same events that were observed in the real Sandy often occur in the simulated storm several hours earlier than actually observed. In Fig. A1b, the simulated wind speed is stronger than the observed wind speed. This difference is partially because the observed wind is a composite field obtained during the flight mission period, whereas the simulated wind is an instantaneous field. The radar reflectivity from the WRF is somewhat higher than the observed radar reflectivity (Fig. A1a), but this is a feature of the model simulation, as noted by Rogers (2010).

\section{REFERENCES}

Abarca, S. F., and M. T. Montgomery, 2013: Essential dynamics of secondary eyewall formation. J. Atmos. Sci., 70, 3216-3230, https://doi.org/10.1175/JAS-D-12-0318.1.

Abraham, J., J. W. Strapp, C. Fogarty, and M. Wolde, 2004: Extratropical transition of Hurricane Michael: An aircraft investigation. Bull. Amer. Meteor. Soc., 85, 1323-1339, https:// doi.org/10.1175/BAMS-85-9-1323.

Black, M. L., J. F. Gamache, F. D. Marks, C. E. Samsury, and H. E. Willoughby, 2002: Eastern Pacific Hurricanes Jimena of 1991 and Olivia of 1994: The effect of vertical shear on structure and intensity. Mon. Wea. Rev., 130, 2291-2312, https://doi.org/ 10.1175/1520-0493(2002)130<2291:EPHJOA>2.0.CO;2.

Blake, E. S., T. B. Kimberlain, R. J. Berg, J. P. Cangialosi, and J. L. Beven II, 2013: Tropical cyclone report: Hurricane Sandy. National Hurricane Center Rep., 157 pp., https://www.nhc.noaa.gov/ data/tcr/AL182012_Sandy.pdf.

Bui, H. H., R. K. Smith, M. T. Montgomery, and J. Peng, 2009: Balanced and unbalanced aspects of tropical cyclone intensification. Quart. J. Roy. Meteor. Soc., 135, 1715-1731, https:// doi.org/10.1002/qj.502.

Evans, C., and R. E. Hart, 2008: Analysis of the wind field evolution associated with the extratropical transition of Bonnie (1998). Mon. Wea. Rev., 136, 2047-2065, https://doi.org/10.1175/ 2007MWR2051.1.

— , and Coauthors, 2017: The extratropical transition of tropical cyclones. Part I: Cyclone evolution and direct impacts. Mon. Wea. Rev., 145, 4317-4344, https://doi.org/10.1175/MWR-D17-0027.1.

Evans, J. L., and B. E. Prater-Mayes, 2004: Factors affecting the posttransition intensification of Hurricane Irene (1999). Mon. Wea. Rev., 132, 1355-1368, https://doi.org/10.1175/15200493(2004)132<1355:FATPIO > 2.0.CO;2.

_ J. M. Arnott, and F. Chiaromonte, 2006: Evaluation of operational model cyclone structure forecasts during extratropical transition. Mon. Wea. Rev., 134, 3054-3072, https:// doi.org/10.1175/MWR3236.1.

Fujibe, F., and N. Kitabatake, 2007: Classification of surface wind fields of tropical cyclones at landfall on the Japan main islands. 
J. Meteor. Soc. Japan, 85, 747-765, https://doi.org/10.2151/ jmsj.85.747.

Galarneau, T. J., C. A. Davis, and M. A. Shapiro, 2013: Intensification of Hurricane Sandy (2012) through extratropical warm core seclusion. Mon. Wea. Rev., 141, 4296-4321, https:// doi.org/10.1175/MWR-D-13-00181.1.

Jones, S. C., and Coauthors, 2003: The extratropical transition of tropical cyclones: Forecast challenges, current understanding, and future directions. Wea. Forecasting, 18, 1052-1092, https:// doi.org/10.1175/1520-0434(2003)018<1052:TETOTC >2.0.CO;2.

Keyser, D., M. J. Reeder, and R. J. Reed, 1988: A generalization of Petterssen's frontogenesis function and its relation to the forcing of vertical motion. Mon. Wea. Rev., 116, 762-780, https://doi.org/ 10.1175/1520-0493(1988)116<0762:AGOPFF $>2.0 . \mathrm{CO} ; 2$.

Klein, P. M., P. A. Harr, and R. L. Elsberry, 2000: Extratropical transition of western North Pacific tropical cyclones: An overview and conceptual model of the transformation stage. Wea. Forecasting, 15, 373-395, https://doi.org/10.1175/15200434(2000)015<0373:ETOWNP>2.0.CO;2.

- - - and - 2002: Extratropical transition of western North Pacific tropical cyclones: Midlatitude and tropical cyclone contributions to reintensification. Mon. Wea. Rev., 130, 2240-2259, https://doi.org/10.1175/1520-0493(2002)130<2240: ETOWNP $>2.0 . \mathrm{CO} ; 2$.

Knapp, K. R., and Coauthors, 2011: Globally gridded satellite observations for climate studies. Bull. Amer. Meteor. Soc., 92, 893-907, https://doi.org/10.1175/2011BAMS3039.1.

Kwon, I.-H., and H.-B. Cheong, 2010: Tropical cyclone initialization with a spherical high-order filter and an idealized threedimensional bogus vortex. Mon. Wea. Rev., 138, 1344-1367, https://doi.org/10.1175/2009MWR2943.1.

, - - H.-G. Kang, H.-J. Han, and J.-R. Park, 2010: Structure change of Typhoon Nari (2007) in the weakening stage. Asia-Pac. J. Atmos. Sci., 46, 327-340, https://doi.org/10.1007/ s13143-010-1006-z.

Leroux, M.-D., M. Plu, D. Barbary, F. Roux, and P. Arbogast, 2013: Dynamical and physical processes leading to tropical cyclone intensification under upper-level trough forcing. J. Atmos. Sci., 70, 2547-2565, https://doi.org/10.1175/JAS-D-12-0293.1.
Merrill, R. T., 1993: Tropical cyclone structure. Global guide to tropical cyclone forecasting, World Meteorological Organization Tech. Doc. WMO/TD-560, 2.1-2.60.

Nguyen, L. T., and J. Molinari, 2012: Rapid intensification of a sheared, fast-moving hurricane over the Gulf Stream. Mon. Wea. Rev., 140, 3361-3378, https://doi.org/10.1175/MWR-D11-00293.1.

Quinting, J. F., M. M. Bell, P. A. Harr, and S. C. Jones, 2014: Structural characteristics of T-PARC Typhoon Sinlaku during its extratropical transition. Mon. Wea. Rev., 142, 1945-1961, https://doi.org/10.1175/MWR-D-13-00306.1.

Reasor, P. D., M. D. Eastin, and J. F. Gamache, 2009: Rapidly intensifying Hurricane Guillermo (1997). Part I: Lowwavenumber structure and evolution. Mon. Wea. Rev., 137, 603-630, https://doi.org/10.1175/2008MWR2487.1.

Rogers, R. F., 2010: Convective-scale structure and evolution during a high-resolution simulation of tropical cyclone rapid intensification. J. Atmos. Sci., 67, 44-70, https://doi.org/10.1175/ 2009JAS3122.1.

Shin, J. H., and D.-L. Zhang, 2017: The impact of moist frontogenesis and tropopause undulation on the intensity, size and structural changes of Hurricane Sandy (2012). J. Atmos. Sci., 74, 893-913, https://doi.org/10.1175/JAS-D15-0362.1.

Smith, R. K., and M. T. Montgomery, 2015: Toward clarity on understanding tropical cyclone intensification. J. Atmos. Sci. 72, 3020-3031, https://doi.org/10.1175/JAS-D-15-0017.1.

— - _ - and N. V. Sang, 2009: Tropical cyclone spinup revisited. Quart. J. Roy. Meteor. Soc., 135, 1321-1335, https://doi.org/ 10.1002/qj.428.

Thorncroft, C. D., and S. C. Jones, 2000: The extratropical transitions of Hurricanes Felix and Iris in 1995. Mon. Wea. Rev., 128, 947-972, https://doi.org/10.1175/1520-0493(2000) $128<0947$ :TETOHF $>2.0 . \mathrm{CO} ; 2$.

Yau, M. K., Y. Liu, D.-L. Zhang, and Y. Chen, 2004: A multiscale numerical study of Hurricane Andrew (1992). Part VI: Smallscale inner-core structures and wind streaks. Mon. Wea. Rev., 132, 1410-1433, https://doi.org/10.1175/1520-0493(2004) 132<1410:AMNSOH $>2.0 . \mathrm{CO} ; 2$ 